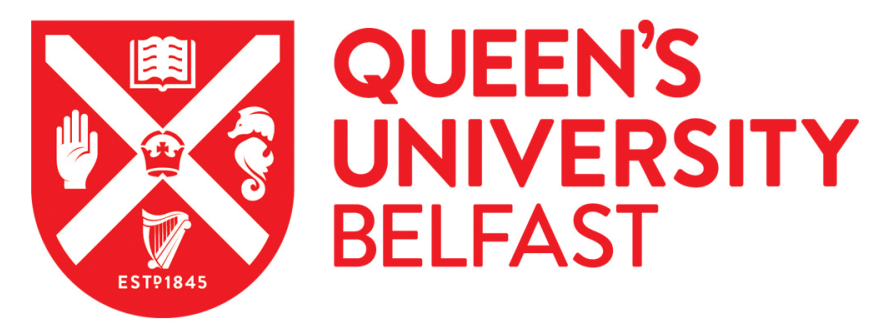

\title{
Comparing the orthogonal and homotopy functor calculi
}

Barnes, D., \& Eldred, R. (2016). Comparing the orthogonal and homotopy functor calculi. Journal of Pure and Applied Algebra, 220(11), 3650-3675. https://doi.org/10.1016/j.jpaa.2016.05.005

\section{Published in:}

Journal of Pure and Applied Algebra

\section{Document Version:}

Peer reviewed version

Queen's University Belfast - Research Portal:

Link to publication record in Queen's University Belfast Research Portal

\section{Publisher rights}

(c) 2016, Elsevier

This manuscript version is made available under the CC-BY-NC-ND 4.0 license http://creativecommons.org/licenses/by-nc-nd/4.0/ , which permits distribution and reproduction for non-commercial purposes, provided the author and source are cited.

\section{General rights}

Copyright for the publications made accessible via the Queen's University Belfast Research Portal is retained by the author(s) and / or other copyright owners and it is a condition of accessing these publications that users recognise and abide by the legal requirements associated with these rights.

Take down policy

The Research Portal is Queen's institutional repository that provides access to Queen's research output. Every effort has been made to ensure that content in the Research Portal does not infringe any person's rights, or applicable UK laws. If you discover content in the Research Portal that you believe breaches copyright or violates any law, please contact openaccess@qub.ac.uk. 


\title{
Comparing the orthogonal and homotopy functor calculi
}

\author{
David Barnes $\quad$ Rosona Eldred
}

\begin{abstract}
Goodwillie's homotopy functor calculus constructs a Taylor tower of approximations to $F$, often a functor from spaces to spaces. Weiss's orthogonal calculus provides a Taylor tower for functors from vector spaces to spaces. In particular, there is a Weiss tower associated to the functor $V \mapsto F\left(S^{V}\right)$, where $S^{V}$ is the one-point compactification of $V$.

In this paper, we give a comparison of these two towers and show that when $F$ is analytic the towers agree up to weak equivalence. We include two main applications, one of which gives as a corollary the convergence of the Weiss Taylor tower of $\mathrm{BO}$. We also lift the homotopy level tower comparison to a commutative diagram of Quillen functors, relating model categories for Goodwillie calculus and model categories for the orthogonal calculus.
\end{abstract}

Keywords: calculus of functors; Goodwillie calculus; orthogonal calculus; enriched categories; model structures; spectra.

\section{Contents}

\begin{tabular}{lll}
\hline 1 & Introduction & 1 \\
\hline
\end{tabular}

2 Background $2 \mathbf{2}$

2.1 Homotopy functor calculus and orthogonal calculus . . . . . . . . . . . . . . . . . . . . 2

2.2 The categories of functors . . . . . . . . . . . . . . . . . . . . . . . . . 3

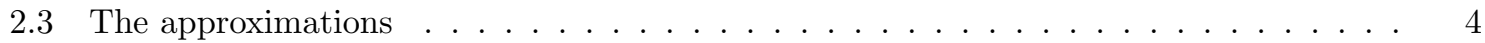

2.4 The homogeneous functors . . . . . . . . . . . . . . . . . . . . . . . . . . . . . . 5

2.5 Connectivity and analyticity . . . . . . . . . . . . . . . . . . . . . . . . . 8

3 Equivalence of the Weiss and Goodwillie tower under $S^{*}$

3.1 Homogeneous functors and restriction . . . . . . . . . . . . . . . . . . . 10

3.2 Restriction of excisive functors are polynomial . . . . . . . . . . . . . . . . . . . . . . . 11

3.3 Agreement of towers for restricted analytic functors . . . . . . . . . . . . . . . . . . . 12

4 Application: Orthogonally weak analytic functors 14

5 Application: Model Category Comparison 15

5.1 The functor $S^{*} \ldots \ldots \ldots \ldots \ldots$

5.2 Comparisons between categories of spectra . . . . . . . . . . . . . . . . . . . . . . 19

5.3 Comparisons between the intermediate categories . . . . . . . . . . . . . . . . . . . . . 21

5.4 Squares 1 and 2 commute . . . . . . . . . . . . . . . . . . . . . . . 23

5.5 Square 3 commutes . . . . . . . . . . . . . . . . . . . . . . . . . . . 26

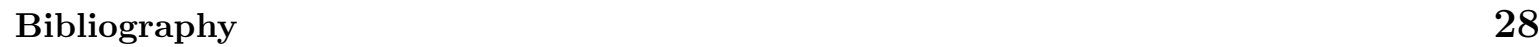




\section{Introduction}

Goodwillie's calculus of homotopy functors, developed originally in [Goo90, Goo91, Goo03, is a method of studying equivalence-preserving functors, motivated by applications to Waldhausen's algebraic $K$-theory of a space. A family of related theories grew out of this work; our focus is on the homotopy functor calculus and the orthogonal calculus of Weiss [Wei95, the latter of which was developed to study functors from real inner-product spaces to topological spaces, such as $B O(V)$ and $T O P(V)$.

The model categorical foundations for the homotopy functor calculus and the orthogonal calculus may be found in Biedermann-Chorny-Röndigs [BCR07], Biedermann-Röndigs [BR13] and Barnes-Oman[BO13] and, most recently, the prequel to this paper, Barnes-Eldred [BE14]. In [BE14], we re-work the classification results of Goodwillie so as to resemble that of the orthogonal calculus. In this paper, we use this similarity to give a formal comparison between the tower arising from orthogonal calculus and that arising from Goodwillie's calculus of homotopy functors. Allusions to such a comparison have existed as folk results for some time, (for example, it is central to [ADL08]). In more general terms, this paper will make it easier to use the two forms of calculus together and transfer calculations between them. For example, the convergence results of Section 4 follow from transferring statements about the Goodwillie tower of a functor to the corresponding Weiss tower.

We start with a background section, Section 2, with necessary definitions and results from the calculi. Section 3 contains a functor level comparison of the theories, with main results as follows. Let $F$ be a functor from based topological spaces to based topological spaces. Then we can consider the functor $V \mapsto F\left(S^{V}\right)$, where $S^{V}$ is the one point compactification of $V$ (a finite dimensional real inner product space). We call this functor from the category of vector spaces and isometries to based topological spaces the restriction of $F$. Proposition 3.1 shows that the restriction of an $n$-homogeneous functor (in the sense of Goodwillie) gives an $n$ homogeneous functor (in the sense Weiss). Similarly, Proposition 3.2 shows that restriction sends $n$-excisive functors to $n$-polynomial functors. We can then consider the restriction of the Goodwillie tower of $F$ and the Weiss tower associated to the functor $V \mapsto F\left(S^{V}\right)$. Theorem 3.5 shows that when $F$ is analytic, these two towers agree.

From these results, we obtain two applications. Firstly, we show in Section 4 that the Weiss tower of the functor $V \mapsto B O(V)$ converges to $B O(V)$ when $V$ has dimension at least 2 (Corollary 4.2). This convergence was claimed without proof in Arone [Aro02, p.13]. This result follows from the more general result we establish (Theorem 4.1) that if the 1st (unstable) derivative of a functor has a kind of analyticity, so does the functor. Secondly, we lift the comparisons of the two forms of calculus to a commutative diagram of model categories and Quillen pairs, see Figure 4 of Section 5. This diagram compares the model structures of $n$-excisive functors, $n$-polynomial functors, $n$-homogeneous functors (of both kinds) and the categories of spectra that classify homogeneous functors.

Acknowledgements Part of this work was completed while the first author was supported by an EPSRC grant (EP/M009114/1), and the latter author by the Humboldt Prize of Michael Weiss. The authors would like to thank Michael Weiss for motivating discussions on 
Section 4 and Greg Arone and Tom Goodwillie for numerous helpful conversations.

\section{Background}

In this section we introduce the orthogonal calculus of Weiss and the homotopy functor calculus of Goodwillie. For the sake of the comparison, we use compatible model category versions of these calculi, namely work of Barnes-Oman [BO13] for orthogonal calculus and Barnes-Eldred [BE14] for the homotopy functor calculus.

We are only interested in the based versions of these two forms of calculus. All spaces are based; we let Top denote the category of based (compactly generated, weak Hausdorff) topological spaces. We omit the customary subscript of $*$ to denote based spaces to allow us to use this space for the subscripts describing various model structures.

\subsection{Homotopy functor calculus and orthogonal calculus}

In either of our two settings, the input $F$ will be a functor from some small category to based spaces. The output will be a tower of functors of the same type as $F$. That is, for each $n \geqslant 0$ there will be a fibration sequence $D_{n} F \rightarrow \mathbb{P}_{n} F \rightarrow \mathbb{P}_{n-1} F$, which can be arranged as below.

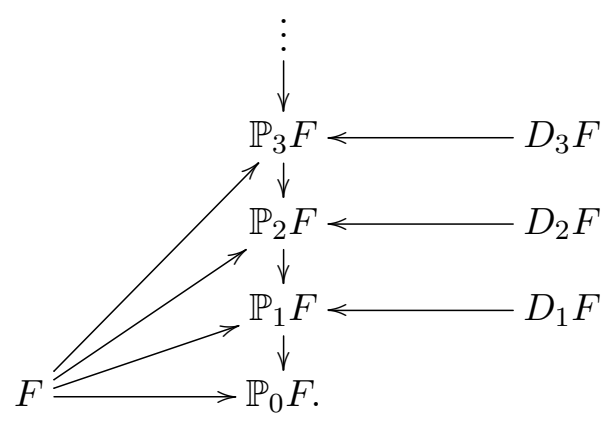

The functors $\mathbb{P}_{n} F$ (which for the homotopy functor calculus are called $P_{n} F$ and for the orthogonal calculus, $\mathbb{T}_{n} F$ ) have a kind of $n$-polynomial property, and for nice functors, the inverse limit of the tower, denoted $\mathbb{P}_{\infty} F$, is equivalent to $F$. We think of each $\mathbb{P}_{n} F$ as an ' $n^{\text {th }}$-approximation' to $F$, moreover $\mathbb{P}_{n} F$ can be recognised as a fibrant replacement of $F$ in some suitable model category. The layers of the tower, $D_{n} F$, are then analogous to purely- $n$-polynomial functors - called $n$-homogeneous.

In each case there is a classification theorem giving the homogeneous functors in terms of some form of spectra. Furthermore, the spectrum corresponding to $D_{n} F$ can be calculated without first constructing $\mathbb{P}_{n} F$ and $\mathbb{P}_{n-1} F$.

For each theory, we will give a definition of the relevant notion of $n$-polynomialness, a construction of the approximations $\mathbb{P}_{n} F$ and a direct construction of spectrum corresponding to the functors $D_{n} F$ and a classification theorem. 


\subsection{The categories of functors}

We introduce the necessary basic categories of functors studied in the homotopy functor calculus and the orthogonal calculus.

For $\mathcal{G}$ a topological group we let $\mathcal{G} \circlearrowleft \mathbb{T}$ op denote the category of based topological spaces with $\mathcal{G}$-action (that fixes the basepoint) and equivariant maps.

Definition 2.1 Let $\mathcal{W}$ be the category of finite based $C W$-complexes, which is enriched over the category of based topological spaces. Let $\mathcal{J}$ be the category of finite dimensional real inner product spaces with morphisms the isometries. Let $\mathcal{J}_{0}$ be the category with the same objects as $\mathcal{J}$, but with morphism spaces given by $\mathcal{J}_{0}(U, V)=\mathcal{J}(U, V)_{+}$. The category $\mathcal{J}_{0}$ is also enriched over Top.

We note that $\mathcal{J}_{0}(V, V)=O(V)_{+}$, and that $\mathcal{J}_{0}(U, V)=*$ whenever the dimension of $U$ is larger than the dimension of $V$.

Definition 2.2 Let $\mathcal{W}$ Top and $\mathcal{J}_{0}$ Top denote the categories of enriched functors from $\mathcal{W}$ and $\partial_{0}$ to Top.

Since both $\mathcal{J}_{0}$ and $\mathcal{W}$ are skeletally small, $\mathcal{W}$ Top and $\mathcal{J}_{0}$ Top have all small limits and colimits, constructed objectwise.

Lemma 2.3 All functors $F$ in $\mathcal{W}$ Top are reduced homotopy functors. That is, $F$ preserves weak equivalences and $F(*)$ is equal to *, the one point space.

Proof. Given any $A$ and $B$ in $\mathcal{W}$, the composite $A \wedge B \cong A \wedge \mathcal{W}\left(S^{0}, B\right) \rightarrow \mathcal{W}(A, A \wedge B)$ induces a map $F(A) \wedge B \rightarrow F(A \wedge B)$. If $f, g: A \rightarrow A^{\prime}$ are maps in $\mathcal{W}$ which are homotopic, then we can construct a homotopy between $F(f)$ and $F(g)$ using the above map in the case $B=[0,1]_{+}$. Since every weak homotopy equivalence in $\mathcal{W}$ is a homotopy equivalence, we see that every object of $\mathcal{W}$ Top preserves weak equivalences.

It also follows that $F(*) \simeq *$. We proceed to show equality: let $0_{X}: X \rightarrow X$ denote the constant map which sends $X$ to the basepoint of $X$. The functor $F$ is enriched over based spaces, so $F\left(0_{X}\right)=0_{F(X)}$. Set $X=*$, then $\operatorname{Id}_{F(*)}=F\left(\operatorname{Id}_{*}\right)$, which is equal to $F\left(0_{*}\right)=0_{F(*)}$. Hence $F(*)$ is $*$.

Definition 2.4 For $A$ and $B$ in $\mathcal{W}$, the map $F(A) \wedge B \rightarrow F(A \wedge B)$ constructed in the previous proof is called the assembly map.

\subsection{The approximations}

We specify the $n$-polynomial properties that occur in the homotopy functor calculus and the orthogonal calculus.

Let $\mathcal{P}(\underline{n})$ denote the poset of subsets of $\{1, \ldots, n\} ; \mathcal{P}_{0}(\underline{n})$ denotes the non-empty subsets and $\mathcal{P}^{1}(\underline{n})$ is all but the final set. 
Definition 2.5 A functor $F \in \mathcal{W T}$ Top is said to be $n$-excisive if for any $X$ the map (induced by the inclusion of $\varnothing$ in the poset of subsets of $\underline{n}$ )

$$
F(X) \longrightarrow \operatorname{holim}_{S \in \mathscr{P}_{0}(\underline{n+1})} F(S * X)=\left(T_{n} F\right)(X)
$$

is a weak homotopy equivalence for each $X$ in $\mathcal{W}$ (* denotes the topological join).

An $n$-cube $X$ in a category $\mathcal{C}$ is a functor $X: \mathcal{P}(\underline{n}) \longrightarrow \mathcal{C}$.

Definition 2.6 We say an $n$-cube $X$ is cartesian if $X(\varnothing) \stackrel{\simeq}{\longrightarrow} \operatorname{holim}_{\mathcal{P}_{0}(\underline{n})} X$. Dually, $X$ is cocartesian if $\operatorname{hocolim}_{\mathcal{P}^{1}(\underline{n}) \stackrel{\simeq}{\longrightarrow}} \mathcal{X}(\underline{n})$. Thus a cartesian 2-cube is a homotopy pullback square and a cocartesian 2-cube is a homotopy pullback squares.

An $n$-cube is strongly cocartesian if every sub-2-cube is cocartesian.

The above definition of $n$-excisive is equivalent to the more standard statement of about a functor taking strongly cocartesian $(n+1)$-cubes to cartesian $(n+1)$-cubes, see [BE14, Lemma 3.13] or [BR13, Lemma 5.9]. A functor is 1-excisive if and only if it takes homotopy pushouts to homotopy pullbacks.

Definition 2.7 For $E \in \mathcal{J}_{0}$ Top define

$$
\tau_{n} E(V)=\operatorname{holim}_{0 \neq U \subset \mathbb{R}^{n+1}} E(U \oplus V)
$$

We say that $E$ is $n$-polynomial if the map

$$
\rho_{E}^{n}(V): E(V) \longrightarrow \tau_{n} E(V)
$$

induced by the maps $E(V) \rightarrow E(U \oplus V)$ is a weak homotopy equivalence for each inner product space $V$.

Definition 2.8 We define $\mathbb{T}_{n} E=$ hocolim $\tau_{n}^{k} E$ for $E \in \mathcal{J}_{0} \mathbb{T}$ Top. Similarly, we define $P_{n} F=$ hocolim $\mathrm{T}_{n}^{k} F$ for $F \in \mathcal{W} \mathbb{T}$ op. We call $\mathbb{T}_{n}$ the $n$-polynomial approximation functor and we call $P_{n}$ the $n$-excisive approximation functor.

The justification for calling these functors approximations comes from the following result, see [Goo03, Theorem 1.8] and [Wei95, Theorem 6.3].

Proposition 2.9 If $F^{\prime}$ is $n$-excisive, then any map $F \rightarrow F^{\prime}$ factors (up to homotopy) over the natural map $F \rightarrow P_{n} F$. If $E^{\prime}$ is n-polynomial, then any map $E \rightarrow E^{\prime}$ factors (up to homotopy) over the natural map $E \rightarrow \mathbb{T}_{n} E$.

The following result is [Goo91, Proposition 3.2] and [Wei95, Proposition 5.4].

Proposition 2.10 Every $(n-1)$-polynomial functor is $n$-polynomial. Every $(n-1)$-excisive functor is $n$-excisive.

Combining these two results gives canonical (up to homotopy) maps $P_{n} F \rightarrow P_{n-1} F$ and $\mathbb{T}_{n} E \rightarrow \mathbb{T}_{n-1} E$ for any $F \in \mathcal{W} \mathbb{T}$ op and any $E \in \mathcal{J}_{0} \mathbb{T}$ op. 


\subsection{The homogeneous functors}

We define $n$-homogeneous functors in each setting and give the classification results of Goodwillie and Weiss.

Definition 2.11 For $E \in \mathcal{J}_{0} \mathbb{T}$ op, we define $D_{n}^{W} E$ to be the homotopy fibre of $\mathbb{T}_{n} E \rightarrow \mathbb{T}_{n-1} E$ (the $W$ stands for Weiss). For $F \in \mathcal{W}$ Top, we define $D_{n}^{G} F$ to be the homotopy fibre of $P_{n} F \rightarrow P_{n-1} F$ (the $G$ stands for Goodwillie).

Definition 2.12 We say that a functor $F \in \mathcal{W} T$ op is $n$-homogeneous if it is $n$-excisive and $P_{n-1} F$ is objectwise contractible. We say that a functor $E \in \mathcal{J}_{0}$ Top is $n$-homogeneous if it is $n$-polynomial and $\mathbb{T}_{n-1} E$ is objectwise contractible.

Since $P_{n-1}$ and $\mathbb{T}_{n-1}$ commute with homotopy fibres, the functors $D_{n}^{G} F$ and $D_{n}^{W} E$ are $n$-homogeneous.

The next result classifies the homogeneous functors (in either setting) in terms of spectra with group actions. For details, see [Goo03, Sections 2-5] and Wei95, Theorem 7.3].

Theorem 2.13 The full subcategory of $n$-homogeneous functors inside $\mathrm{Ho}(\mathcal{W}$ Top) is equivalent to the homotopy category of spectra with $\Sigma_{n}$-action. Given a spectrum $\Theta_{F}$ with $\Sigma_{n}$ action the functor below is an $n$-homogeneous functor of $\mathcal{W}$ Top.

$$
X \mapsto \Omega^{\infty}\left(\left(\Theta_{F} \wedge X^{\wedge n}\right) / h \Sigma_{n}\right)
$$

The full subcategory of $n$-homogeneous functors inside $\mathrm{Ho}_{0}\left(\mathcal{J}_{0} \mathbb{T} \mathrm{To}\right)$ is equivalent to the homotopy category of spectra with $O(n)$-action. Given a spectrum $\Psi_{E}$ with $O(n)$-action the functor below is an $n$-homogeneous functor of $\mathcal{J}_{0} \mathbb{T}$ Top.

$$
V \mapsto \Omega^{\infty}\left(\left(\Psi_{E} \wedge S^{\mathbb{R}^{n} \otimes V}\right) / h O(n)\right)
$$

We now elaborate on how, given an $n$-homogeneous functor $E \in \mathcal{J}_{0} \mathbb{T}$ op or an $n$-homogeneous functor $F \in \mathcal{W} \mathbb{T}$ op, one obtains a spectrum $\Psi_{E}$ or $\Theta_{F}$ such that

$$
E(V) \simeq \Omega^{\infty}\left(\left(\Psi_{E} \wedge S^{\mathbb{R}^{n} \otimes V}\right) / h O(n)\right) \quad \text { or } \quad F(X) \simeq \Omega^{\infty}\left(\left(\Theta_{F} \wedge X^{\wedge n}\right) / h \Sigma_{n}\right) .
$$

We begin with the orthogonal calculus setting. Recall that we denote by $\mathcal{J}$ the category of finite dimensional real inner product spaces with morphisms the isometries. Define a vector bundle over $\mathcal{J}(U, V)$, for $U, V \in \mathcal{J}$ by

$$
\gamma_{n}(U, V)=\left\{(f, x) \mid f \in \mathcal{J}(U, V), x \in \mathbb{R}^{n} \otimes(V-f(U))\right\}
$$

where $V-f(U)$ denotes the orthogonal complement of the image of $U$ under the isometry $f$. The total space has a natural action of $O(n)$ due to the $\mathbb{R}^{n}$ factor. We then let $\mathcal{J}_{n}(U, V):=$ $T \gamma_{n}(U, V)$, the associated Thom space. This is the cofibre in the sequence:

$$
\begin{aligned}
& \underset{2 \|}{S \gamma_{n}(U, V)_{+}} \quad \longrightarrow \quad \underset{2 \|}{D \gamma_{n}(U, V)_{+}} \quad \longrightarrow \quad T \gamma_{n}(U, V) \\
& \{(f, x) \mid\|x\|=1\} \quad\{(f, x) \mid\|x\| \leqslant 1\}
\end{aligned}
$$


Recall that $T\left(\mathbb{R}^{n} \rightarrow *\right)=S^{n}$ and $T(X=X)=X_{+}$, for compact $X$. In particular, if we let $n=0$, we see that $\mathcal{J}_{0}(U, V)=\mathcal{J}(U, V)_{+}$as already defined.

There is a natural composition when looking at the bundles

$$
\begin{aligned}
& \gamma_{n}(V, W) \quad \times \quad \gamma_{n}(U, V) \quad \longrightarrow \quad \gamma_{n}(U, W) \\
& (g, y) \quad(f, x) \quad \mapsto \quad\left(g \circ f, y+\left(\mathbb{R}^{n} \otimes g\right) x\right)
\end{aligned}
$$

where $\left(\mathbb{R}^{n} \otimes g\right): \mathbb{R}^{n} \otimes(V-f(U)) \rightarrow \mathbb{R}^{n} \otimes W$. This composition induces unital and associative maps

$$
\mathcal{\partial}_{n}(V, W) \wedge \mathcal{\partial}_{n}(U, V) \rightarrow \mathcal{\partial}_{n}(U, W),
$$

which are $O(n)$-equivariant and functorial in the inputs.

Definition 2.14 We define an $O(n) \circlearrowleft \mathbb{T}$ op-enriched category $\mathcal{J}_{n}$, whose objects are finite dimensional real inner product spaces and morphism spaces are given by $\mathfrak{J}_{n}(U, V)$.

Define $O(n) \ltimes\left(\mathcal{J}_{n}\right.$ Top $)$ to be the category of $O(n) \circlearrowleft \mathbb{T}$ op-enriched functors from $\mathcal{J}_{n}$ to $O(n) \circlearrowleft \mathbb{T o p}$.

The map $i_{n}: \mathcal{J}_{0}(U, V) \rightarrow \mathcal{J}_{n}(U, V), f \mapsto(f, 0)$ induces a map of Top-enriched categories $\partial_{0} \rightarrow \partial_{n}$ (where we ignore the $O(n)$-action).

The map of enriched categories $i_{n}: \mathcal{\partial}_{0} \rightarrow \mathcal{\partial}_{n}$ induces a (restriction) functor $\operatorname{Res}_{0}^{n}:=i_{n}^{*}$ from $O(n) \ltimes\left(\mathcal{J}_{n} \mathbb{T}\right.$ op $)$ to $\mathcal{J}_{0} \mathbb{T}$ op, which also forgets the $O(n)$-action. We now introduce a functor $\operatorname{Ind}_{0}^{n}$ from $\mathcal{J}_{0}$ Top to $O(n) \ltimes\left(\mathcal{J}_{n} \mathbb{T}\right.$ op). Here we take the definition from [BO13, Section 4], rather than the closely-related [Wei95, Proposition 2.1].

Definition 2.15 For $E \in \mathcal{J}_{0}$ Top, we define the stable $n^{\text {th }}$-derivative of $E$ to be

$$
\operatorname{Ind}_{0}^{n} E \in O(n) \ltimes\left(\mathcal{J}_{n} \mathbb{T o p}\right) \quad \text { where } \operatorname{Ind}_{0}^{n} E(V)=\operatorname{Nat}_{\mathcal{J}_{0} \mathbb{T o p}}\left(\mathcal{J}_{n}(V,-), E\right)
$$

where the $O(n)$-action on $\operatorname{Ind}_{0}^{n} E(V)$ is induced from the $O(n)$ action on $\mathcal{J}_{n}(V,-)$.

For $E \in \mathcal{J}_{0} \mathbb{T}$ op, we define the unstable $n^{\text {th }}$-derivative of $E$ to be $\operatorname{Res}_{0}^{n} \operatorname{Ind}_{0}^{n} E \in \mathcal{J}_{0} \mathbb{T}_{\mathrm{op}}$.

It is common to abuse notation slightly and write $\operatorname{Res}_{0}^{n} \operatorname{Ind}_{0}^{n}$ as simply $\operatorname{Ind}_{0}^{n}$ when the context is clear.

Remark 2.16 The functor $\operatorname{Res}_{0}^{n} \operatorname{Ind}_{0}^{n} E \in \mathcal{J}_{0}$ Top is usually considered in unstable model categories such as the objectwise model structure or the $n$-polynomial model structure. Whereas $\operatorname{Ind}_{0}^{n} E \in O(n) \ltimes\left(\mathcal{J}_{n} \mathbb{T o p}\right)$ is only considered in a stable model structure, hence the choice of names.

The category $O(n) \ltimes\left(\mathcal{J}_{n}\right.$ Top $)$ has a stable model structure which makes it Quillen equivalent to the category spectra with an $O(n)$-action. We will elaborate on this further in Section 5 . For now, we simply give the following (imprecise) theorem.

Theorem 2.17 The $O(n)$-spaces $\operatorname{Ind}_{0}^{n} E(V)$ for $V \in \mathcal{J}_{0}$ define a spectrum $\Psi_{E}$ with $O(n)$ action. Furthermore, the $n$-homogeneous functor constructed from $\Psi_{E}$ by Theorem 2.13 is equivalent to $D_{n}^{W} E$. 
Now we turn to the homotopy functor calculus equivalent and consider a more direct way to construct the spectrum corresponding to $D_{n}^{G} F$.

Definition 2.18 For $F \in \mathcal{W T}$ Top and an $n$-tuple of spaces in $\mathcal{W},\left(X_{1}, \ldots, X_{n}\right)$, the $n^{\text {th }}-$ cross effect of $F$ at $\left(X_{1}, \ldots, X_{n}\right)$ is the space

$$
\operatorname{cr}_{n}(F)\left(X_{1}, \ldots, X_{n}\right)=\operatorname{Nat}\left(\bigwedge_{l=1}^{n} \mathcal{W}\left(X_{l},-\right), F\right)
$$

We alter the cross effect construction to obtain a similar construction to $\operatorname{Ind}_{0}^{n}$ from the orthogonal calculus, see [BE14, Section 6].

Definition 2.19 The category $\mathcal{W}_{n}$ is a $\Sigma_{n} \circlearrowleft \mathbb{T}$ Top-enriched category with objects the finite dimensional $C W$-complexes and morphism spaces given by

$$
\mathcal{W}_{n}(A, B)=\bigwedge_{k=1}^{n} \mathcal{W}(A, B)
$$

The group $\Sigma_{n}$ acts on this space by permuting factors. Let $\Sigma_{n} \ltimes\left(\mathcal{W}_{n}\right.$ Top $)$ be the category of $\Sigma_{n} \circlearrowleft \mathbb{T}$ Top-enriched functors from $\mathcal{W}_{n}$ to $\Sigma_{n} \circlearrowleft \mathbb{T}$ op. We may then define the stable $n$th derivative of $F$ to be

$$
\operatorname{diff}_{n}(F) \in \Sigma_{n} \ltimes\left(\mathcal{W}_{n} \mathbb{T o p}\right) \quad \text { where } \operatorname{diff}_{n}(F)(X)=\operatorname{Nat}\left(\mathcal{W}_{n}(X,-), F\right) .
$$

Pre-composing $\operatorname{diff}_{n}(F)$ with the diagonal map $\mathcal{W}(X, Y) \rightarrow \mathcal{W}_{n}(X, Y)$ and forgetting the $\Sigma_{n}$-action yields a functor in WTop. We call this the unstable $n$th derivative of $F$ and also denote it by $\operatorname{diff}_{n}(F)$.

Remark 2.20 The unstable $n$th derivative of $F \in \mathcal{W T}$ Top is also given by by pre-composing $\operatorname{cr}_{n}(F)$ with the diagonal map. We usually consider the unstable derivative in unstable model structures on $\mathcal{W}$ Top, such as the objectwise or n-excisive model structure. Whereas we only consider $\Sigma_{n} \ltimes\left(\mathcal{W}_{n} \mathbb{T}\right.$ op) with a stable model structure, see Proposition 5.20, hence the choice of names.

In [BE14] is also shown that (when equipped with a suitable model structure) $\Sigma_{n} \ltimes\left(\mathcal{W}_{n}\right.$ Top) is Quillen equivalent to spectra with an $\Sigma_{n}$-action. We will elaborate on this further in Section 5. For now we simply give the following (imprecise) theorem.

Theorem 2.21 The $\Sigma_{n}$-spaces $\operatorname{diff}_{n} F(X)$ define a spectrum $\Theta_{F}$ with $\Sigma_{n}$-action. Furthermore, the $n$-homogeneous functor constructed from $\Theta_{F}$ by Theorem 2.13 is $D_{n}^{G} F$.

\subsection{Connectivity and analyticity}

Notions of convergence and how well a functor is approximated by its tower of $n$-excisive (respectively, $n$-polynomial) approximations rely on the following definitions of stably $n$ excisive and $\rho$-analyticity, as well as their orthogonal analogues. The results for orthogonal calculus are a re-phrasing and expansion on results found in Wei98. We will make use of these especially in Section 3 . 
Roughly speaking, if $F \in \mathcal{W}$ Top is stably $n$-excisive then it takes strong cocartesian $(n+1)$ cubes to almost cartesian cubes. We say that a cube if $k$-cartesian if $X(\varnothing) \stackrel{\simeq}{\longrightarrow} \operatorname{holim}_{\mathcal{P}_{0}(\underline{n})} X$ is $k$-connected.

Definition 2.22 [Goo91, Defn 4.1] $F \in \mathcal{W}$ Top is stably $n$-excisive or satisfies stable $n^{\text {th }}$ order excision if the following holds for some numbers $c$ and $\kappa$ :

$$
\begin{aligned}
& E_{n}(c, \kappa): \text { If } \mathscr{X}: \mathscr{P}(S) \rightarrow \mathscr{C} \text { is any strongly co-cartesian }(n+1) \text {-cube such that } \\
& \forall s \in S \text { the map } \mathscr{X}(\varnothing) \rightarrow \mathscr{X}(s) \text { is } k_{s} \text {-connected and } k_{s} \geqslant \kappa \text {, then the } \\
& \quad \text { diagram } F(\mathscr{X}) \text { is }\left(-c+\sum k_{s}\right) \text {-cartesian. }
\end{aligned}
$$

It is usual to instead consider the following property which is satisfied for some $\rho$ by many important functors.

Definition 2.23 [Goo91, Defn 4.2] $F \in \mathcal{W T}$ Top is $\rho$-analytic if there is some number $q$ such that $F$ satisfies $E_{n}(n \rho-q, \rho+1)$ for all $n \geqslant 1$.

Remark 2.24 By definition, $\rho$-analytic functors are stably $n$-excisive for all $n$.

One of the main consequences of $\rho$-analyticity is the following, which we formally define as it is a relevant notion for orthogonal calculus as well; for functors in JTop, we use dimension instead of connectivity.

Definition 2.25 We say that $F \in \mathcal{W} T$ Top is weakly $\rho$-analytic if for any space of connectivity at least $\rho, F(X) \stackrel{\simeq}{\longrightarrow} P_{\infty} F(X)$. If $F$ is $\rho$-analytic, then $F$ is weakly $\rho$-analytic.

We say that $E \in \mathcal{J}_{0} \mathbb{T}$ op is weakly $\rho$-analytic if for any vector space of dimension at least $\rho, E(X) \stackrel{\simeq}{\longrightarrow} \mathbb{T}_{\infty} E(X)$.

Definition 2.26 Goo03, Definition 1.2] A map $u: F \rightarrow G$ in $\mathcal{W}$ Top satisfies $O_{n}(c, \kappa)$ if $\forall k \geqslant \kappa \forall X \in C$ with $X k$-connected, $u_{X}: F(X) \rightarrow G(X)$ is $(-c+(n+1) k)$-connected.

We say that $F$ and $G$ agree to order $n$ (via $u$ ) if $u$ satisfies $O_{n}(c, \kappa)$ for some $c, \kappa$. We also call $u$ an agreement to order $n$.

The orthogonal analogue translates connectivity into dimension:

Definition 2.27 Let $p: F \rightarrow G$ be a morphism in $\mathcal{J}_{0}$ Top. Suppose there exists a $b$ such that $p_{W}: F W \rightarrow G W$ is $(-b+(n+1) \operatorname{dim}(W))$-connected for all $W \in \mathcal{J}_{0}$ of dimension bigger than some $\kappa$. Then we say that $F$ and $G$ agree up to orth-order $n$ via $p$ or $p$ is an orth-order- $n$ agreement between $F$ and $G$.

Proposition 2.28 Goo03, Proposition 1.5] If F is stably $n$-excisive, then

$1 P_{n} F$ is $n$-excisive and

$2 F$ agrees with $P_{n} F$ to order $n\left(\right.$ via $p_{n}: F \rightarrow P_{n} F$ )

Lemma 2.29 If $F$ is stably $n$-excisive, then $S^{*} p_{n}: S^{*} F \rightarrow P_{n}\left(S^{*} F\right)$ is an orth-order-n agreement. 
Proof. The connectivity of $S^{V}$ is $\operatorname{dim}(V)-1$ and $F$ is stably $n$-excisive, so Goo03, Proposition 1.5] implies that $p_{n, S^{V}}: F\left(S^{V}\right) \rightarrow P_{n} F\left(S^{V}\right)$ has connectivity

$$
\left.\left(-c+(n+1) \operatorname{conn}\left(S^{V}\right)\right)\right)=(-c-(n+1)+(n+1) \operatorname{dim}(V))
$$

for some $c$ whenever $\operatorname{conn}\left(S^{V}\right)=\operatorname{dim}(V)-1$ is larger than some $\kappa$. Taking $-b=-c+(n+1)$, we conclude that $S^{*} p_{n}: S^{*} F \rightarrow P_{n}\left(S^{*} F\right)$ is an orth-order- $n$ agreement.

Proposition 2.30 [Goo03, Proposition 1.6] Let $u: F \rightarrow G$ be a map in WTop. If $F$ and $G$ agree to order $n$ (via $u$ ) then the induced map $P_{n} u: P_{n} F \rightarrow P_{n} G$ is an objectwise weak equivalence.

Proposition 2.30 has the following orthogonal calculus analogue.

Proposition 2.31 Let $F, G$ be functors in Jo $_{0}$ Top. If $p: F \rightarrow G$ is an orth-order-n agreement, then $\mathbb{T}_{n}(p): \mathbb{T}_{n} F \rightarrow \mathbb{T}_{n} G$ is an objectwise weak equivalence.

Proof. A simple alteration to the proof of [Wei98, Lemma e.3] shows that if the map $p_{W}$ : $F(W) \rightarrow G(W)$ is $(-b+(n+1) \operatorname{dim}(W))$-connected for all $W \in \mathcal{J}_{0}$ with $\operatorname{dim}(W)>\kappa$, then $\left(\tau_{n} p\right)_{W}(-b+(n+1) \operatorname{dim}(W)+1)$-connected whenever $\operatorname{dim}(W)>\kappa-1$.

Since $\mathbb{T}_{n}(p)=\mathbb{T}_{n}\left(\tau_{n}^{\kappa} p\right)$ the result [Wei98, Lemma e.7] implies that $\mathbb{T}_{n}(p)_{W}$ is an equivalence for all $W \in \mathcal{J}_{0}$.

Remark 2.32 Notice that since the connectivity of $S^{V}$ is $\operatorname{dim}(V)-1$, if $F \in \mathcal{W}$ Top is $\rho$-analytic, we expect the functor $\left(V \mapsto F\left(S^{V}\right)\right) \in \mathcal{J}_{0}$ Top to be (weakly) $(\rho+1)$-analytic. See Corollary 3.6 and Example 3.7 .

\section{Equivalence of the Weiss and Goodwillie tower under $S^{*}$}

We can view $\mathcal{J}_{0}$ via $V \mapsto S^{V}$ as a subcategory of $\mathcal{W}$ (see [MMSS01, Remark 4.7]). Let $F$ be a homotopy functor, i.e. $F \in \mathcal{W}$ Top. We then call $V \mapsto F\left(S^{V}\right)=\left(S^{*} F\right)(V)$ its restriction to the image of $\mathcal{J}_{0}$ in $\mathcal{W}$. In this section we will show that the restriction of the Goodwillie tower of a $\rho$-analytic functor is weakly equivalent to the Weiss tower of its restriction. Definitions of the terms $\rho$-analytic and stably $n$-excisive may be found in Section 2.5. Heuristically, these are both connectivity assumptions on what a functor does to strongly cocartesian cubes.

\subsection{Homogeneous functors and restriction}

We begin by considering $n$-homogeneous functors and use the classification results of Goodwillie and Weiss to conclude that the restriction of an $n$-homogeneous functor in $\mathcal{W}$ Top is $n$-homogeneous in $\mathcal{J}_{0}$ Top.

Proposition 3.1 If a functor $F$ in WTop is $n$-homogeneous in the sense of Goodwillie, then $S^{*} F \in \mathcal{J}_{0} \mathbb{T}$ op is $n$-homogeneous in the sense of Weiss. 
Proof. Let $F$ be an $n$-homogeneous functor in $\mathcal{W}$ Top. Then, by Theorem 2.13 and Theorem 2.21, from $F$ we can obtain $\Theta_{F}$, a spectrum with an action of $\Sigma_{n}$ such that

$$
F(A) \simeq \Omega^{\infty}\left(A^{\wedge n} \wedge \Theta_{F}\right) / h \Sigma_{n} \quad, \forall A \in \mathcal{W}
$$

Using the (derived) change of groups functor, we can construct a spectrum with an action of $O(n)$ from $\Theta_{F}$ :

$$
O(n)_{+} \wedge \Sigma_{n}^{L} \Theta_{F}=O(n)_{+} \wedge \Sigma_{n}\left(\left(E \Sigma_{n}\right)_{+} \wedge \Theta_{F}\right)
$$

Using Theorem 2.13, we obtain an $n$-homogeneous functor $F^{\prime}$ in $\mathcal{J}_{0} \mathbb{T}$ op

$$
F^{\prime}(V)=\Omega^{\infty}\left(S^{n V} \wedge O(n)_{+} \wedge \Sigma_{n}\left(\left(E \Sigma_{n}\right)_{+} \wedge \Theta_{F}\right)\right) / h O(n)
$$

Let $i^{*}$ denote the forgetful functor from $O(n)$-spaces to $\Sigma_{n}$-spaces. If $X$ is a space with $\Sigma_{n}$-action and $Y$ is a space with $O(n)$-action, we have an isomorphism of $O(n)$-spaces $\left(O(n)_{+} \wedge \Sigma_{n} X\right) \wedge Y \rightarrow O(n)_{+} \wedge \Sigma_{n}\left(X \wedge i^{*} Y\right)$ given by $[g, x, y] \mapsto\left[g, x, g^{-1} y\right]$. This isomorphism extends to the level of spectra, to give an isomorphism

$$
F^{\prime}(V) \cong \Omega^{\infty}\left(O(n)_{+} \wedge \Sigma_{n}\left(i^{*} S^{n V} \wedge\left(\left(E \Sigma_{n}\right)_{+} \wedge \Theta_{F}\right)\right) / h O(n) .\right.
$$

The action of $O(n)$ on $\left(O(n)_{+} \wedge \Sigma_{n}\left(i^{*} S^{n V} \wedge\left(\left(E \Sigma_{n}\right)_{+} \wedge \Theta_{F}\right)\right)\right.$ is free: the only fixed point of each level of the spectrum is the basepoint, since $\left(E \Sigma_{n}\right)_{+}$is a free $\Sigma_{n}$-space. Thus, taking $O(n)$-homotopy orbits is the same as taking strict orbits. We hence have a series of isomorphisms as below.

$$
\begin{aligned}
F^{\prime}(V) & \cong \Omega^{\infty}\left(O(n)_{+} \wedge \Sigma_{n}\left(i^{*} S^{n V} \wedge\left(\left(E \Sigma_{n}\right)_{+} \wedge \Theta_{F}\right)\right)\right) / O(n) \\
& \cong \Omega^{\infty}\left(i^{*} S^{n V} \wedge\left(\left(E \Sigma_{n}\right)_{+} \wedge \Theta_{F}\right)\right) / \Sigma_{n} \\
& \cong \Omega^{\infty}\left(i^{*} S^{n V} \wedge \Theta_{F}\right) / h \Sigma_{n} \\
& \simeq F\left(S^{V}\right)
\end{aligned}
$$

We have shown that $S^{*} F$ is objectwise weakly equivalent to $F^{\prime}$ and hence it is an $n$ homogeneous functor in the sense of Weiss.

Let $\mathrm{Ho}(\mathcal{W}$ Top) be the homotopy category of $\mathcal{W}$ Top, where a weak equivalence is a objectwise weak equivalence (see Proposition 5.1). We then define $n-h o m o g \operatorname{Ho}(\mathcal{W} T$ op) to be the full subcategory of Ho WTop with objects the $n$-homogeneous functors; we define n-homog $\operatorname{Ho}\left(\mathcal{J}_{0} \mathbb{T}\right.$ op) analogously. For $\mathcal{G}$ a group, let $\operatorname{Ho}(\mathcal{G} \circlearrowleft \mathrm{Sp})$ be the homotopy category of spectra with an action of $\mathcal{G}$.

Then the previous result shows that the derived functor $R S^{*}$ of $S^{*}$ is well defined on the categories of $n$-homogeneous functors. Furthermore, it shows that Figure 1 is commutative, where the vertical arrows are from the classification statements of Weiss and Goodwillie.

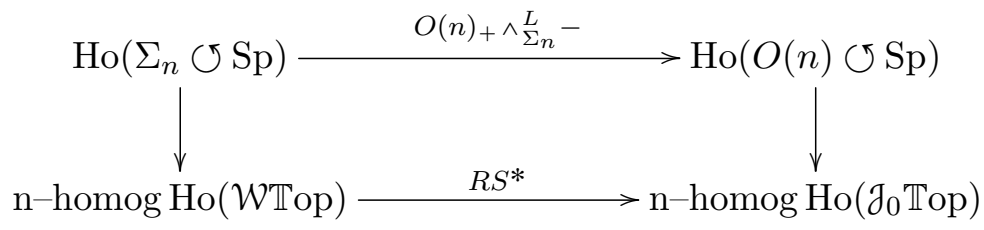

Figure 1: The homotopy level diagram 


\subsection{Restriction of excisive functors are polynomial}

In this section, we prove that the restriction of an $n$-excisive functor is $n$-polynomial.

Proposition 3.2 For $F$ a homotopy functor, the map $w_{n}: S^{*}\left(P_{n} F\right) \rightarrow \mathbb{T}_{n}\left(S^{*}\left(P_{n} F\right)\right)$ is an objectwise weak equivalence. Equivalently, restriction to $\mathcal{J}_{0}$ of an $n$-excisive functor yields an $n$-polynomial functor.

Proof. We work by induction. If $n=0$ then $S^{*} P_{0} F=*$, which is 0 -polynomial in $\mathcal{J}_{0}$ Top. Now we assume the result holds for $n-1$. By [Goo03, Lemma 2.2], there is a fibre sequence with $R_{n} F \in \mathcal{W}$ Top an $n$-homogeneous in the sense of Goodwillie:

$$
P_{n} F \longrightarrow P_{n-1} F \stackrel{\rho}{\longrightarrow} R_{n} F
$$

By Proposition 3.1, we know $S^{*} R_{n} F$ is $n$-homogeneous in the orthogonal calculus. It follows that $\operatorname{Ind}_{0}^{n+1} R_{n} F\left(S^{V}\right)$ is trivial. By inductive hypothesis, $S^{*} P_{n-1} F$ is $(n-1)$-polynomial, and thus $n$-polynomial (by Proposition 2.10). Hence, we may apply [Wei95, Lemma 5.5] to

$$
S^{*} \rho: S^{*}\left(P_{n-1} F\right) \longrightarrow S^{*}\left(R_{n} F\right)
$$

to conclude that its homotopy fibre is $n$-polynomial in the orthogonal calculus. Since homotopy fibres are constructed objectwise, the homotopy fibre is $S^{*}\left(P_{n} F\right)$.

Remark 3.3 Proposition 3.2 implies that if $F$ satisfies $F(X) \stackrel{\simeq}{\longrightarrow} \operatorname{holim}_{k \in \mathscr{P}_{0}([n])} F(k * X)$ for all $X \in \mathcal{W}$, then $S^{*} F$ satisfies $F\left(S^{V}\right) \stackrel{\simeq}{\longrightarrow} \operatorname{holim}_{0 \neq U \subset \mathbb{R}^{n+1}} F\left(S^{V \oplus U}\right)$ for all $V \in \mathcal{J}_{0}$. This can be thought of as a kind of enriched cofinality.

\subsection{Agreement of towers for restricted analytic functors}

It is observed in Goo03 that when $F$ is stably n-excisive, $P_{n} F$ is, up to natural equivalence, the only $n$-excisive functor that agrees to $n^{\text {th }}$ order with $F$. The uniqueness of $P_{n} F$ for these functors implies more than just $n$-polynomialness of $S^{*}\left(P_{n} F\right)$ - we can also say that $S^{*}\left(P_{n} F\right)$ is the $n$-polynomial approximation of $S^{*} F$. If we assume the stronger property of $\rho$-analyticity for $F$ (which implies that $F$ is stably $n$-excisive for all $n$ ), then we can conclude that the tower $\left\{\mathbb{T}_{n}\left(S^{*} F\right)\right\}_{n \geqslant 0}$ is equivalent to the tower $\left\{S^{*}\left(P_{n} F\right)\right\}_{n \geqslant 0}$.

Proposition 3.4 If $F$ is a stably n-excisive homotopy functor, the following map is a weak homotopy equivalence for all $V \in \mathcal{J}_{0}$.

$$
\mathbb{T}_{n}\left(S^{*} p_{n}\right)(V): \mathbb{T}_{n}\left(S^{*} F\right)(V) \longrightarrow \mathbb{T}_{n}\left(S^{*} P_{n} F\right)(V)
$$

Thus the $n$-polynomial approximation of $S^{*} F$ is given by the map $S^{*} F \rightarrow S^{*}\left(P_{n} F\right)$.

Proof. For any $F \in \mathcal{W}$ Top there is a commutative square as below. Proposition 3.2 implies that the right hand vertical map is a weak equivalence.

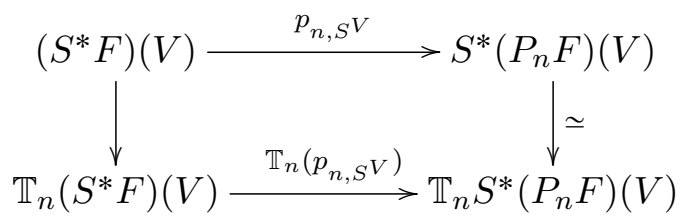


Assume that $F \in \mathcal{W}$ Top is stably $n$-excisive, then by [Goo03, Proposition 1.5], the map $p_{n}: F \rightarrow P_{n} F$ is an agreement to order $n$ (Definition 2.26). By Lemma 2.29, we also know that the restriction to $\mathcal{J}_{0}$

$$
S^{*} p_{n}: S^{*} F \longrightarrow S^{*}\left(P_{n} F\right)
$$

is an orth-order- $n$ agreement (Definition 2.27). Then, applying Proposition 2.31, we conclude that

$$
\mathbb{T}_{n}\left(S^{*} p_{n}\right): \mathbb{T}_{n}\left(S^{*} F\right) \longrightarrow \mathbb{T}_{n}\left(S^{*}\left(P_{n} F\right)\right)
$$

is an objectwise equivalence. The commutative square then shows that the $n$-polynomial approximation to $S^{*} F, S^{*} F \rightarrow \mathbb{T}_{n} S^{*} F$, is weakly equivalent to $S^{*} F \rightarrow S^{*}\left(P_{n} F\right)$.

To extend to an equivalence of towers, not just of the $n^{\text {th }}$ level for a fixed $n$, we consider the stronger notion of $\rho$-analytic.

Theorem 3.5 Consider $F$ a $\rho$-analytic homotopy functor for some $\rho$ and $S^{*} F$ its restriction to $\mathcal{J}_{0}$. Then the Weiss tower of $S^{*} F$ is equivalent to the restriction to $\mathcal{J}_{0}$ of the Goodwillie tower of $F$.

Proof. Since $\rho$-analytic functors are stably $n$-excisive for all $n$ (Remark 2.24), we have a commutative diagram as below. Moreover, the horizontal maps are objectwise weak equivalences by Propositions 3.2 and 3.4 .

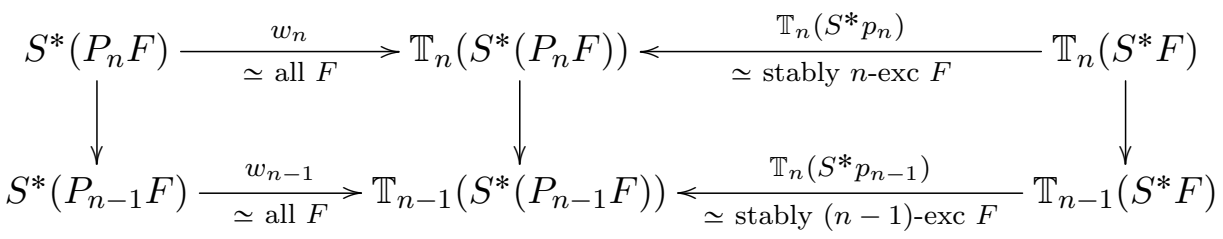

It follows that $S^{*}\left(D_{n}^{G} F\right)$ is objectwise weakly equivalent to $D_{n}^{W} S^{*} F$. Furthermore, by Proposition 3.1. if $\Theta_{F}$ is the spectrum with $\Sigma_{n}$-action that corresponds to $D_{n}^{G} F$ (by Goodwillie's classification, see Theorem 2.13, then $O(n)_{+} \wedge_{\Sigma_{n}}^{L} \Theta_{F}$ is the spectrum corresponding to $D_{n}^{W} S^{*} F$ (by Weiss's classification).

While $S^{*} P_{n} F$ is $n$-polynomial, in general there is no reason for it to be the $n$-polynomial approximation to $S^{*} F$. Consequently, for non-analytic functors $F$, the two towers $\left\{S^{*} P_{n} F\right\}_{n \geqslant 0}$ and $\left\{\mathbb{T}_{n}\left(S^{*} F\right)\right\}_{n \geqslant 0}$ do not need to be equivalent under $S^{*} F$.

Corollary 3.6 If $F \in \mathcal{W}$ Top is $\rho$-analytic, $S^{*} F$ agrees to orth-order-n with $\mathbb{T}_{n}\left(S^{*} F\right)$ for all $n$. Moreover, $S^{*} F$ is weakly $(\rho+1)$-analytic. That is, for $V$ at least dimension $(\rho+1)$, we have that $S^{*} F(V) \stackrel{\simeq}{\longrightarrow} \mathbb{T}_{\infty}\left(S^{*} F\right)(V)=\operatorname{hocolim}_{n}\left(\mathbb{T}_{n} S^{*} F\right)(V)$.

Proof. Since $F$ is $\rho$-analytic, we have weak equivalences for all $n$

$$
S^{*}\left(P_{n} F\right) \stackrel{\simeq}{\longrightarrow} \mathbb{T}_{n}\left(S^{*}\left(P_{n} F\right)\right) \stackrel{\simeq}{\longleftarrow} \mathbb{T}_{n}\left(S^{*} F\right)
$$

and we have the commutative diagram of objects of $\mathcal{J}_{0}$ Top as in Figure 2. We let $q_{n}: P_{n} \rightarrow$ $P_{n-1}$ and $v_{n}: \mathbb{T}_{n} \rightarrow \mathbb{T}_{n-1}$ be the natural transformations arising from Proposition 2.10 . 


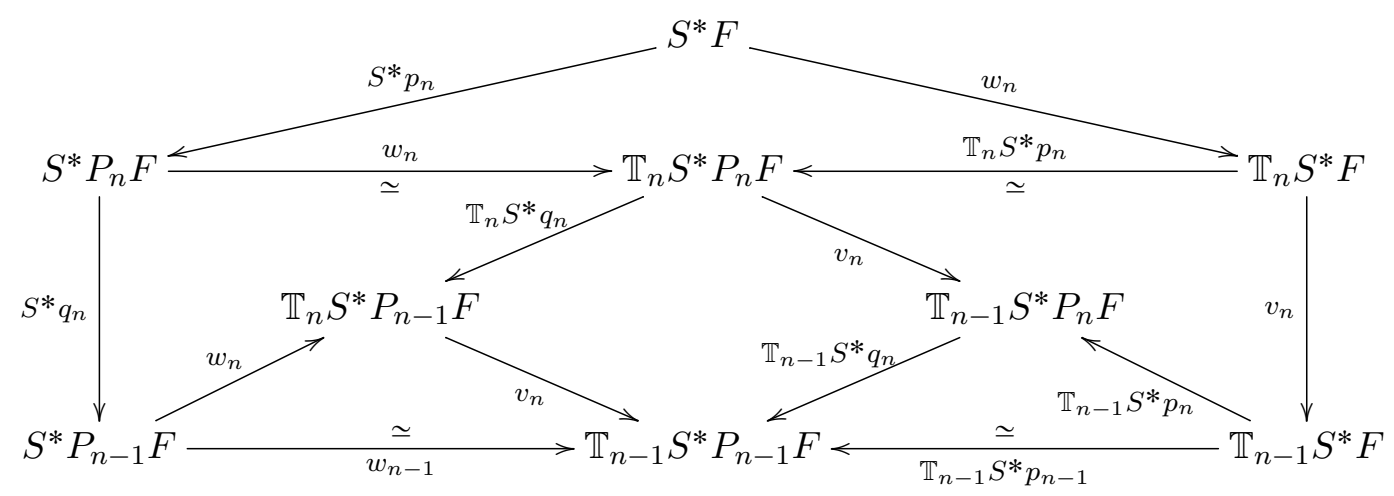

Figure 2: Commutativity of the tower maps

The above figure implies that we have a commutative diagram of homotopy limits as below, with the maps from $S^{*} F$ are weak equivalences only when $\operatorname{dim}(V) \geqslant \rho+1$ (so that $S^{V}$ is in $F$ 's radius of convergence).

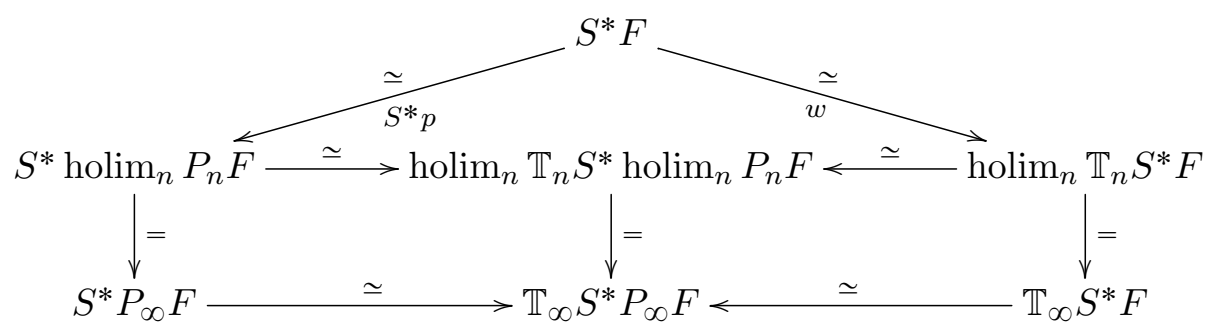

In particular, $S^{*} F(V) \stackrel{\simeq}{\longrightarrow} \mathbb{T}_{\infty} S^{*} F(V)$ for all $V$ of dimension larger than $\rho$, as desired.

Example 3.7 The identity functor $\operatorname{Id}_{\mathbb{T} \text { op }} \in \mathcal{W} \mathbb{T}$ op is 1-analytic. Therefore, $V \mapsto S^{V}$ (which is equal to $S^{*} \mathrm{Id}_{\mathbb{T o p}}$ ) is weakly 2-analytic, by Corollary 3.6.

\section{Application: Orthogonally weak analytic functors}

In this section, we show that one can obtain weak analyticity results for functors in $\mathcal{J}_{0}$ Top whose unstable first derivative is known already to be analytic. Our immediate application is that the Weiss tower of $V \mapsto B O(V)$ converges to $V \mapsto B O(V)$ when $\operatorname{dim}(V) \geqslant 2$ and similarly for $V \mapsto B U(V)$ for $V$ of dimension $\geqslant 1$.

Theorem 4.1 Let $E$ be a functor in $\mathcal{J}_{0}$ Top. Assume that its unstable first orthogonal derivative, the functor $V \mapsto \operatorname{Ind}_{0}^{1} E(V)$ in $\mathcal{J}_{0}$ Top, is weakly $\rho$-analytic. Assume, moreover, that for $V$ of dimension at least $\rho, E(V)$ is path-connected. Then $E$ is itself weakly $\rho$-analytic.

Corollary 4.2 The functor $V \mapsto B O(V)$ is weakly 2 -analytic and the functor $V \mapsto B U(V)$ is weakly 1-analytic. 
This follows immediately from Theorem 4.1, using the facts $\operatorname{Ind}_{0}^{1} B O(V) \simeq S^{V}$ Wei95, Example 2.7], $\operatorname{Ind}_{0}^{1} B U(V) \simeq \Sigma S^{V}$ and $V \mapsto S^{V}$ is weakly 2-analytic by Example 3.7. Recall that for $\mathcal{G}$ a topological group $B \mathcal{G}=E \mathcal{G} / \mathcal{G}$ is path connected.

This particular analyticity bound matches well with observed behaviour of $B O$ - that it does not behave well on $V$ with dimension less than 2 (see, for instance, Proposition 3.4 and 3.5 of Reis-Weiss [RW15]).

Remark 4.3 This is a formalization of the comment of Arone in [Aro02, p.13] that the Taylor tower of $B O$ converges.

Proof. (of Theorem 4.1) It suffices to argue with a skeleton of $\mathcal{J}_{0}$, that is, with $\mathbb{R}^{n}$ instead of arbitrary $V$ of dimension $n$. In the following, we let $E\left(\mathbb{R}^{\infty}\right)$ denote the homotopy colimit over $k$ of $E\left(\mathbb{R}^{k}\right)$ for $E \in \mathcal{J}_{0} \mathbb{T}$ op.

Recall that $E$ is weakly $\rho$-analytic if and only if $E(V) \stackrel{\sim}{\longrightarrow} \mathbb{T}_{\infty} E(V)$ for all $V$ of dimension at least $\rho$.

For $E$ a functor in $\mathcal{J}_{0}$ Top there is a homotopy fibre sequence

$$
\operatorname{Ind}_{0}^{1} E(V) \longrightarrow E(V) \longrightarrow E(V \oplus \mathbb{R})
$$

by [Wei95, Proposition 2.2]. Since $\mathbb{T}_{\infty}$ preserves fibre sequences, the natural transformation $E \rightarrow \mathbb{T}_{\infty} E$ yields a map of fibre sequences as in Figure 3 .

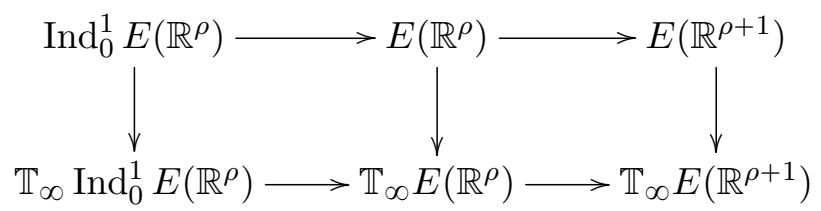

Figure 3: map of fibre sequences

We now show that the right hand square is cartesian: compare the long exact sequence of homotopy groups for (1) the homotopy fibre sequence that is the top of Figure 3 and (2) the homotopy fibre sequence that is

$$
\operatorname{Ind}_{0}^{1} E\left(\mathbb{R}^{\rho}\right) \longrightarrow Q_{\rho} \longrightarrow E\left(\mathbb{R}^{\rho+1}\right)
$$

Here, $Q_{\rho}$ is the homotopy pullback of

$$
\mathbb{T}_{\infty} E\left(\mathbb{R}^{\rho}\right) \longrightarrow \mathbb{T}_{\infty} E\left(\mathbb{R}^{\rho+1}\right) \longleftarrow E\left(\mathbb{R}^{\rho+1}\right) .
$$

The five lemma applied to the long exact sequences of homotopy groups associated to (1) and (2) and a simple diagram chase for $\pi_{0}$ (using path-connectedness of $E\left(\mathbb{R}^{\rho}\right)$ and $E\left(\mathbb{R}^{\rho+1}\right)$ ) shows that $E\left(\mathbb{R}^{\rho}\right) \rightarrow Q_{\rho}$ is a weak homotopy equivalence. Consequently, the right hand square of Figure 3 is cartesian as desired.

We can extend the right hand square of Figure 3 to the right to get a shifted copy of the square. By the same argument, this is also cartesian. We can repeat this process to obtain 


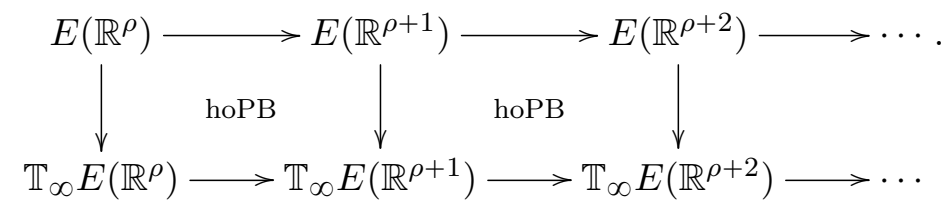

Since the juxtaposition of two cartesian squares is cartesian, for all $n \geqslant \rho$ and $k \geqslant 1$ we have a cartesian square as on the left of the diagram below. Filtered homotopy colimits preserve cartesian squares, hence the square on the right of the diagram below is cartesian.
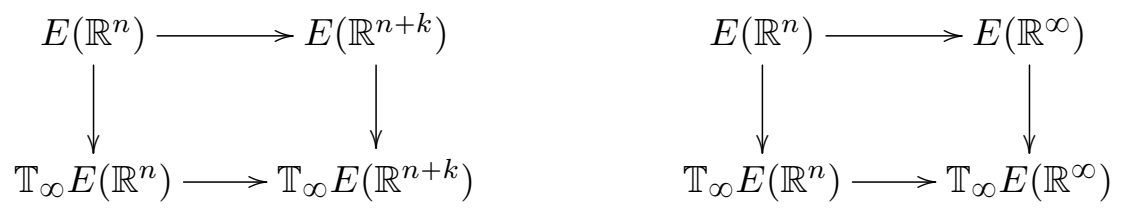

For any $\mathcal{F} \in \mathcal{J}_{0} \mathbb{T}$ op and any $q \geqslant 0$, the map $\mathcal{F}\left(\mathbb{R}^{\infty}\right) \longrightarrow \mathbb{T}_{q} \mathcal{F}\left(\mathbb{R}^{\infty}\right)$ is a weak homotopy equivalence by [Wei95, Lemma 5.14]. That is, the (orthogonal) Taylor tower of $\mathcal{F}$ at $\mathbb{R}^{\infty}$ is constant. Therefore, $\mathcal{F}\left(\mathbb{R}^{\infty}\right) \stackrel{\simeq}{\longrightarrow} \mathbb{T}_{\infty} \mathcal{F}\left(\mathbb{R}^{\infty}\right)$, and in particular, this holds for $\mathcal{F}=E$. Thus the right hand vertical in the right hand square above is a weak homotopy equivalence. Since the square is cartesian, the left hand vertical is a weak homotopy equivalence for any $n \geqslant \rho$ as desired.

\section{Application: Model Category Comparison}

In the previous sections we have examined the relations between the orthogonal calculus and the homotopy functor calculus at the level of homotopy categories. We now lift this to the level of model structures and construct the diagram of model categories and Quillen functors as in Figure 4. We will then show that it commutes, in the sense that certain compositions of functors agree up to natural isomorphism. The notation here has been chosen to match the preceding paper [BE14]. The next few sections introduce the categories and model structures used and then we turn to showing that the squares of the diagram commute. 


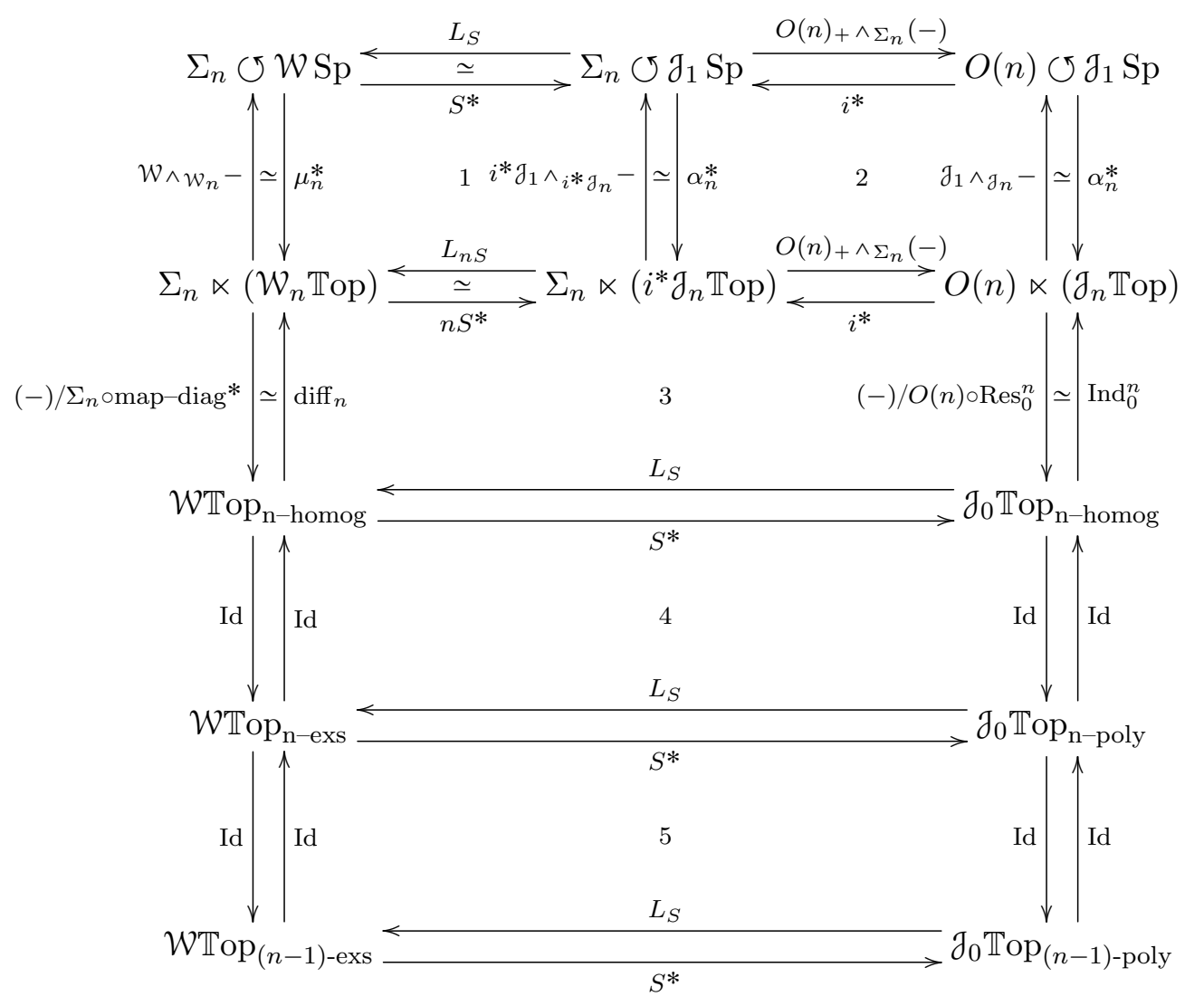

Figure 4: The diagram of model categories

In [BE14] the model categories and adjunctions of the leftmost column are constructed and shown to give a model category version of Goodwillie's calculus of functors (for based spaces). The constructions of that paper are strongly related to the model structures constructed in BR13. That paper considers a more general setting, based around simplicial functors between simplicial model categories.

\subsection{The functor $S^{*}$}

Here we recap the definition of the model categories needed for the two forms of calculus and show that $S^{*}$ can be given the structure of a Quillen functor. Thus squares 4 and 5 of Figure 4 are commutative squares of Quillen functors.

Recall the functor $S$ from $\mathcal{J}_{0}$ to $\mathcal{W}$, which sends $V$ to the one-point compactification $S^{V}$. Given $F \in \mathcal{W}$ Top we can pre-compose with $S$ to obtain $S^{*} F:=F \circ S: \jmath_{0} \rightarrow \mathbb{T}$ op. This functor has a left adjoint, called $L_{S}$, which is given by the formula below.

$$
\left(L_{S} E\right)(A)=\int^{V \in \mathcal{J}_{0}} E(V) \wedge \mathcal{W}\left(S^{V}, A\right)
$$


In [MMSS01], $S$ is called $\mathbb{U}$ and its left adjoint is denoted $\mathbb{P}$.

Proposition 5.1 There are objectwise model structures on WTop and $\mathcal{J}_{0}$ Top whose fibrations and weak equivalences are defined objectwise. They are proper and cofibrantly generated.

For the cross effect (Definition 2.18) to be a right Quillen functor requires more cofibrations than in the objectwise model structure on $\mathcal{W}$ Top. We now specify the extra maps which are needed:

Definition 5.2 Consider the following collection of maps, where $\phi_{\underline{X}, n}$ is defined via the projections which send those factors in $S$ to the basepoint.

$$
\Phi_{n}=\left\{\phi_{\underline{X}, n}: \operatorname{colim}_{S \in \mathcal{P}_{0}(\underline{n})} \mathcal{W}\left(\bigvee_{l \in \underline{n}-S} X_{l},-\right) \longrightarrow \mathcal{W}\left(\bigvee_{l=1}^{n} X_{l},-\right) \mid \underline{X}=\left(X_{1}, \ldots, X_{n}\right), X_{l} \in \text { sk } \mathcal{W}\right\}
$$

We then also define $\Phi_{\infty}=\cup_{n \geqslant 1} \Phi_{n}$.

The cofibre of $\operatorname{Nat}(-, F)\left(\phi_{\underline{X}, n}\right)$ is the cross effect of $F$ at $\underline{X}, \operatorname{cr}_{n}(F)\left(X_{1}, \ldots, X_{n}\right)$; see [BR13, Lemma 3.14].

Definition 5.3 Given $f: A \rightarrow B$ a map of based of spaces and $g: F \rightarrow G$ in $\mathcal{W}$ Top, the pushout product of $f$ and $g, f \square g$, is given by

$$
f \circ g: B \wedge F \bigvee_{A \wedge F} A \wedge G \rightarrow B \wedge G .
$$

where $(B \wedge F)(C)=B \wedge F(C)$.

Recall the generating sets $I_{\mathbb{T} \text { op }}$ and $J_{\mathbb{T} o p}$ for the weak-homotopy equivalence model structure on based topological spaces.

Proposition 5.4 There is a proper, cofibrantly generated model structure on WTop, the cross effect model structure, whose weak equivalences are the objectwise weak homotopy equivalences and whose generating sets are given by

$$
I_{\mathcal{W}_{c r}}=\Phi_{\infty} \square I_{\mathbb{T o p}} \quad \text { and } \quad J_{\mathcal{W}_{c r}}=\Phi_{\infty} \square J_{\mathbb{T o p}} .
$$

Every cross effect fibration is in particular a objectwise fibration.

Proof. See [BE14, Theorem 3.6] and [BO13, Lemma 6.1].

The point of the cross effect model structure is that it allows diff ${ }_{n}$ to be a right Quillen functor, see [BE14, Proposition 6.3]. Now that we have our initial model structures we can show that $S^{*}$ is a right Quillen functor.

Lemma 5.5 The functor $S^{*}$ is a right Quillen functor when $\mathcal{W}$ Top and $\mathcal{J}_{0}$ Top are both equipped with the objectwise model structures. Furthermore it is a right Quillen functor when WTop has the cross effect model structure and JoTop is equipped with the objectwise model structure. 
Proof. Take some (acyclic) fibration $f$ in the objectwise model structure on $\mathcal{W}$ Top. Then $f(A)$ is a (acyclic) fibration of spaces for any $A \in \mathcal{W}$. Hence $f\left(S^{V}\right)$ is a (acyclic) fibration of spaces for any $V \in \mathcal{J}_{0}$. This proves the first statement. For the second, we note that the identity functor is a right Quillen functor from the cross effect model structure to the objectwise model structure, since every cross effect fibration is, in particular, an objectwise fibration and the model structures have the same weak equivalences.

In Square 5 of Figure 4 we are interested in the $n$-excisive and $n$-polynomial model structures, which we introduce below. For the proof, see [BE14, Theorem 3.14] and [BO13, Proposition $6.5]$.

Proposition 5.6 There is an $n$-excisive model structure on WTop which has the same cofibrations as the cross effect model structure and whose weak equivalences are those maps $f$ such that $P_{n} f$ is an objectwise weak equivalence. The fibrant objects are the $n$-excisive functors that are fibrant in the cross effect model structure. A map $f: X \rightarrow Y$ is an nexcisive fibration if and only if it is a cross effect fibration and the square below is cartesian.

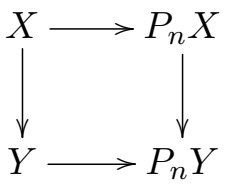

There is an $n$-polynomial model structure on $\mathcal{J}_{0}$ Top which has the same cofibrations as the objectwise model structure and whose weak equivalences are those maps $f$ such that $\mathbb{T}_{n} f$ is a objectwise weak equivalence. The fibrant objects are the $n$-polynomial functors.

Both of these model structures are proper and cofibrantly generated.

The functor $S^{*}$ remains a right Quillen functor with respect to these model structures.

Proposition 5.7 The functor $S^{*}$ is a right Quillen functor between $\mathcal{W}$ Top with the $n$ excisive model structure and $\mathcal{J}_{0}$ Top with the $n$-polynomial model structure.

Proof. We must show that the Quillen pair of the previous lemma induces a Quillen pair between the model categories $\mathcal{W} \mathbb{T}_{\mathrm{n}} \mathrm{p}_{\mathrm{n} \text { exs }}$ and $\mathcal{J}_{0} \mathbb{T}_{\mathrm{op}}$-poly. We know that the functor $S^{*}$ is a right Quillen functor from $\mathcal{W}$ Top to $\mathcal{J}_{0} \mathbb{T}$ op, where both categories have their objectwise model structures. Similarly the identity functor from $\mathcal{W} \mathbb{T}_{0} p_{n-e x s}$ to $\mathcal{W}$ Top (with the objectwise model structure) is a right Quillen functor. The result then follows by [Hir03, Theorem 3.1.6] and Section 3.2, which proves that $S^{*}$ sends $n$-excisive functors to $n$-polynomial functors.

Corollary 5.8 The derived functor of $S^{*}$ from $\mathcal{W} \mathbb{T}_{0 p_{n-e x s}}$ to $\mathcal{J}_{0} \mathbb{T}_{0 p_{n-p o l y}}$ is given by $S^{*} \circ$ $P_{n}$. When restricted to stably $n$-excisive functors, the derived functor is given by $S^{*}$ (by Proposition 3.4).

Remark 5.9 Proposition 3.2 implies that the left adjoint $L_{S}$ takes maps of the form

$$
\delta_{n, U, V}: \operatorname{hocolim}_{0 \neq U \subseteq \mathbb{R}^{n+1}} \mathcal{J}_{0}(U \oplus V,-) \longrightarrow \mathcal{J}_{0}(V,-)
$$


(for $V \in \mathcal{J}_{0}$ ) to $P_{n}$-equivalences in $\mathcal{W}$ Top. That is,

$$
L_{S} \delta_{n, U, V}: \operatorname{hocolim}_{0 \neq U \subseteq \mathbb{R}^{n+1}} \mathcal{W}\left(S^{U} \wedge S^{V},-\right) \longrightarrow \mathcal{W}\left(S^{V},-\right)
$$

is a $P_{n}$-equivalence for all $V \in \mathcal{J}_{0}$ Top.

In Square 4 of Figure 4 we have $n$-homogeneous model structures, which we introduce below. For the details, see [BE14, Theorem 3.18] and [BO13, Proposition 6.9].

Proposition 5.10 There is an n-homogeneous model structure on $\mathcal{W}$ Top which has the same fibrations as the n-excisive model structure and whose weak equivalences are those maps $f$ such that $D_{n}^{G} f$ is an objectwise weak equivalence. The cofibrant-fibrant objects are the $n$-homogeneous functors that are cofibrant-fibrant in the cross effect model structure.

There is an $n$-homogeneous model structure on $\mathcal{J}_{0}$ Top which has the same fibrations as the $n$-excisive model structure and whose weak equivalences are those maps $f$ such that $D_{n}^{W} f$ is an objectwise weak equivalence. The cofibrant-fibrant objects are the $n$-homogeneous functors that are cofibrant in the objectwise model structure.

Both of these model structures are proper, stable and cofibrantly generated.

The functor $S^{*}$ is not just a right Quillen functor for $n$-polynomial functors, but also for $n$-homogeneous.

Theorem 5.11 The functor $S^{*}$ is a right Quillen functor from $\mathcal{W}$ Top (equipped with the $n$ homogeneous model structure) to $\mathcal{J}_{0}$ Top (equipped with the $n$-homogeneous model structure).

Proof. Since the $n$-homogeneous fibrations are precisely the $n$-excisive (or $n$-polynomial) fibrations, $S^{*}$ preserves fibrations. Let $f: X \rightarrow Y$ in $\mathcal{W}$ Top be an acyclic fibration in the $n$-homogeneous model structure. By [BR13, Lemma 6.22] $f$ is an $(n-1)$-excisive fibration. Thus $S^{*} f$ is an $(n-1)$-polynomial fibration and the homotopy fibre of $S^{*} f$ is $(n-1)$ polynomial. The model category $\mathcal{J}_{0} \mathbb{T}_{0} p_{n-h o m o g}$ is stable and $(n-1)$-polynomial functors are trivial in this model structure, hence $S^{*} f$ is a weak equivalence in $\mathcal{J}_{0} \mathbb{T}_{0} p_{n-h o m o g}$.

\subsection{Comparisons between categories of spectra}

Recall from Definition 2.14 that $\partial_{1}$ is an enriched category of finite dimensional real inner product spaces with the space of morphisms the Thom space of the following vector bundle:

$$
\gamma_{1}(U, V)=\{(f, x) \mid f \in \mathcal{J}(U, V), x \in V-f(U)\} .
$$

Definition 5.12 For $\mathcal{G}=\Sigma_{n}$ or $O(n)$ and $\mathcal{D}=\mathcal{J}_{1}$ or $\mathcal{W}, \mathcal{G} \circlearrowleft \mathcal{D}$ Sp denotes the category of $\mathcal{G}$-objects and $\mathcal{G}$-equivariant maps in $\mathcal{D T}$ Top, with the stable model structure. The weak equivalences are those maps which forget to $\pi_{*}$-isomorphisms of non-equivariant fibre spectra.

In each case the notation $\mathrm{Sp}$ is to remind us that the stable model structure is being used. We describe the objects of these model categories as spectra, as they model stable homotopy types in the the corresponding homotopy categories. 
In this section, we compare the three categories of spectra with group action: $\Sigma_{n} \circlearrowleft \mathcal{W} \mathrm{Sp}$, $\Sigma_{n} \circlearrowleft \mathcal{J}_{1} \mathrm{Sp}$ and $O(n) \circlearrowleft \mathcal{J}_{1} \mathrm{Sp}$. In terms of the diagram in Figure 4, this comparison is the top row:

$$
\Sigma_{n} \circlearrowleft \mathcal{W} \mathrm{Sp} \underset{S^{*}}{\stackrel{L_{S}}{\longleftarrow}} \Sigma_{n} \circlearrowleft \mathcal{J}_{1} \mathrm{Sp} \underset{i^{*}}{\stackrel{O(n)_{+} \wedge \Sigma_{n}(-)}{\longleftarrow}} O(n) \circlearrowleft J_{1} \mathrm{Sp}
$$

Recall that an orthogonal spectrum is simply a continuous functor from $\mathcal{J}_{1}$ to $\mathbb{T}$ op and a $\mathcal{W}$ spectrum is a continuous functor from $\mathcal{W}$ to Top. In both cases, we can forget structure to obtain a sequential spectrum: a collection of spaces $\left\{X_{n}\right\}_{n \geqslant 0}$ with maps $X_{n} \wedge S^{1} \rightarrow X_{n+1}$. In the orthogonal case, we set $X_{n}$ to be the functor evaluated at $\mathbb{R}^{n}$, in the $\mathcal{W}$ case we set it to be the functor evaluated at $S^{n}$.

Proposition 5.13 For $\mathcal{G}$ either $\Sigma_{n}$ or $O(n)$, the stable model structure on $\mathcal{G} \circlearrowleft \mathcal{D} \mathrm{Sp}$ is proper, stable and cofibrantly generated.

The generating cofibrations are given by $\mathcal{G}_{+} \wedge \mathcal{D}(d,-) \wedge i$ where $d$ is an element of a skeleton of $\mathcal{D}$ and $i$ is a generating cofibration of based topological spaces. The cofibrant objects are $\mathcal{G}$-free (they have no $\mathcal{G}$-fixed points).

Proof. The non-equivariant stable model structures $\mathcal{W} \mathrm{Sp}$ and $\mathcal{J}_{1} \mathrm{Sp}$ exist by MMSS01, Theorem 9.2]. The equivariant versions exist by applying the transfer argument of Hirschhorn [Hir03, Theorem 11.3.2] to the free functor $\mathcal{G}_{+} \wedge-$ from $\mathcal{D}$ Sp to $\mathcal{G} \circlearrowleft \mathcal{D} \mathrm{Sp}$.

The one-point compactification construction induces a functor of enriched categories $S: \mathcal{J}_{1} \rightarrow$ $\mathcal{W}$. The mapping spaces of $\partial_{1}$ admit the description (see [MM02, Definition 4.1] for more details)

$$
\partial_{1}(U, V)=O(V)_{+} \wedge O(V-U) S^{V-U}
$$

Note that this requires us to choose some preferred inclusion $U \hookrightarrow V$. We want to define a map of spaces $\mathcal{J}_{1}(U, V) \rightarrow \mathcal{W}\left(S^{U}, S^{V}\right)$, it suffices (due to adjointness) to construct a map

$$
O(V)_{+} \wedge O(V-U) S^{V-U} \wedge S^{U} \rightarrow S^{V}
$$

To do so, we simply compose the isomorphism $S^{V-U} \wedge S^{U} \rightarrow S^{V}$ with the action map of $O(V)$ on $S^{V}$.

Hence pre-composition with $S$ is a functor $S^{*}: \mathcal{W T o p} \rightarrow \mathcal{J}_{1}$ Top. This induces a functor between the respective categories of $\Sigma_{n}$-objects and $\Sigma_{n}$-equivariant maps.

Lemma 5.14 The adjoint pair $\left(L_{S}, S^{*}\right)$ is a Quillen equivalence between $\Sigma_{n} \circlearrowleft \mathcal{W}$ Sp and $\Sigma_{n} \circlearrowleft \mathcal{J}_{1} \mathrm{Sp}$.

Proof. The non-equivariant statement is [MMSS01, Theorem 0.1]. The equivariant result follows from the fact that the fibrations (weak equivalences) of both model categories are defined by forgetting to the non-equivariant versions.

We now compare $\Sigma_{n} \circlearrowleft \mathcal{J}_{1}$ Sp and $O(n) \circlearrowleft \mathcal{J}_{1}$ Sp. Given an object in $O(n) \circlearrowleft \mathcal{J}_{1}$ Sp we may forget some of the action and obtain a $\Sigma_{n}$-object. We call this functor $i^{*}$. It has a left adjoint given by $O(n)_{+} \wedge \Sigma_{n}-$. See [MM02, Section 2] for more details (in the terms of that reference, we are using the trivial universe $\mathbb{R}^{\infty}$ for our equivariant spectra). 
Lemma 5.15 The functor $i^{*}$ is a right Quillen functor (with respect to the stable model structures) from $O(n) \circlearrowleft \mathcal{J}_{1} \mathrm{Sp}$ to $\Sigma_{n} \circlearrowleft \mathcal{J}_{1} \mathrm{Sp}$.

Proof. That $i^{*}$ preserves fibrations and weak equivalences is immediate: both classes are defined by forgetting the group actions entirely.

We have now shown that the top row of Figure 4 consists of Quillen functors between model categories.

\subsection{Comparisons between the intermediate categories}

We now move on to the second row of Figure 4, which compares the various intermediate categories.

$$
\Sigma_{n} \ltimes\left(\mathcal{W}_{n} \mathbb{T o p}\right) \underset{n S^{*}}{\longleftarrow} \Sigma_{n} \ltimes\left(i^{*} \partial_{n} \mathbb{T o p}\right) \underset{i^{*}}{\stackrel{O(n)_{+} \Sigma_{n}(-)}{\longleftarrow}} O(n) \ltimes\left(\partial_{n} \mathbb{T o p}\right)
$$

This section is similar to the previous one, but with more complicated enriched categories $\partial_{n}$ and $\mathcal{W}_{n}$, see Definition 2.14 and Definition 2.19.

Definition 5.16 We define $\Sigma_{n} \ltimes\left(\mathcal{W}_{n} \mathbb{T}\right.$ op) as the category of $\Sigma_{n} \circlearrowleft \mathbb{T}$ op-enriched functors from $\mathcal{W}_{n}$ to $\Sigma_{n} \circlearrowleft \mathbb{T}$ op. Similarly, $O(n) \ltimes\left(\mathcal{J}_{n} \mathbb{T} o p\right)$ is the category of $O(n) \circlearrowleft \mathbb{T}$ op-enriched functors from $\partial_{n}$ to $O(n) \circlearrowleft \mathbb{T}$ op. By forgetting structure, we obtain a $\Sigma_{n} \circlearrowleft$ Top-enriched category $i^{*} \mathcal{J}_{n}$. We define $\Sigma_{n} \ltimes\left(i^{*} \mathcal{J}_{n}\right.$ Top) to be the category of $\Sigma_{n} \circlearrowleft \mathbb{T}$ op-enriched functors from $i^{*} \mathcal{J}_{n}$ to $\Sigma_{n} \circlearrowleft \mathbb{T}$ op.

We first compare $\Sigma_{n} \ltimes\left(\mathcal{W}_{n} \mathbb{T}\right.$ op $)$ and $\Sigma_{n} \ltimes\left(i^{*} \mathcal{J}_{n} \mathbb{T}\right.$ op $)$, via an adjunction induced by a map of enriched categories $n S: i^{*} \mathcal{J}_{n} \rightarrow \mathcal{W}_{n}$, which we construct below.

Recall (Definition 2.14) that $\mathcal{J}_{n}(U, V)$ is defined as the Thom space of the vector bundle

$$
\gamma_{n}(U, V)=\left\{(f, x) \mid f \in \mathcal{J}(U, V), x \in \mathbb{R}^{n} \otimes(V-f(U))\right\}
$$

over the space of linear isometries from $U$ to $V, \mathcal{L}(U, V)$. Note that $\mathcal{J}_{0}(U, V)=\mathcal{L}(U, V)_{+}$.

Projection onto factor $l$ (the map from $\mathbb{R}^{n} \rightarrow \mathbb{R}$ via $\left.\left(x_{1}, \ldots x_{n}\right) \mapsto x_{l}\right)$, induces a morphism of vector bundles $\gamma_{n}(U, V) \rightarrow \gamma_{1}(U, V)$. These maps induced a $\Sigma_{n}$-equivariant a map of vector bundles as below, where the map into term $l$ is induced by projection onto factor $l$.

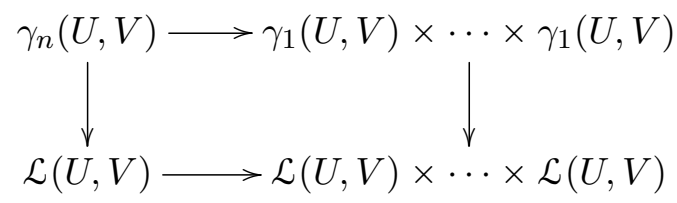

Taking the induced map on Thom spaces gives a $\Sigma_{n}$-equivariant morphism as below, see [Hus94, Chapter 15, Proposition 1.5].

$$
i^{*} \partial_{n}(U, V) \longrightarrow \bigwedge_{k=1}^{n} \partial_{1}(U, V)
$$


Composing this map with the $n$-fold smash of the map $S$ gives a $\Sigma_{n}$-equivariant map

$$
i^{*} \partial_{n}(U, V) \longrightarrow \bigwedge_{k=1}^{n} \mathcal{W}\left(S^{U}, S^{V}\right)
$$

Hence we have a map of $\Sigma_{n} \circlearrowleft \mathbb{T}$ op-categories $i^{*} \mathcal{J}_{n} \rightarrow \mathcal{W}_{n}$, which we call $n S$.

Definition 5.17 Let $F$ be an object of $\Sigma_{n} \ltimes\left(\mathcal{W}_{n} \mathbb{T o p}\right)$, pre-composing $F$ with nS gives a functor $n S^{*} F:=F \circ n S$ from $i^{*} \jmath_{n}$ to $\Sigma_{n} \circlearrowleft \mathbb{T}$ Top. Hence, $n S^{*}$ is a functor from $\Sigma_{n} \ltimes$ $\left(\mathcal{W}_{n} \mathbb{T o p}\right)$ to $\Sigma_{n} \ltimes\left(i^{*} \mathcal{J}_{n} \mathbb{T}\right.$ op $)$. This has a left adjoint given by the formula

$$
\left(L_{n S} E\right)(A)=\int^{V \in \mathcal{J}_{0}} E(V) \wedge \mathcal{W}_{n}\left(S^{V}, A\right) .
$$

As with spectra, we have change of groups adjunctions, which are closely related to the constructions of [MM02, Section 2]. Changing from $\Sigma_{n}$-equivariance to $O(n)$-equivariance gives us an adjunction between $\Sigma_{n} \ltimes\left(i^{*} \mathcal{J}_{n} \mathbb{T}\right.$ op $)$ and $O(n) \ltimes\left(\mathcal{J}_{n} \mathbb{T}\right.$ op $)$.

Definition 5.18 Let $E \in O(n) \ltimes\left(\mathcal{J}_{n} \mathbb{T}\right.$ op), then $E$ has $O(n)$-equivariant structure maps

$$
E_{U, V}: \mathcal{J}_{n}(U, V) \rightarrow \mathbb{T o p}(E(U), E(V)) .
$$

We can forget structure to obtain a $\Sigma_{n}$-equivariant map

$$
i^{*} E_{U, V}: i^{*} \mathcal{J}, n(U, V) \rightarrow i^{*} \mathbb{T o p}(E(U), E(V))=\mathbb{T o p}\left(i^{*} E(U), i^{*} E(V)\right) .
$$

This gives a functor $i^{*}: O(n) \ltimes\left(\mathcal{J}_{n} \mathbb{T o p}\right) \rightarrow \Sigma_{n} \ltimes\left(i^{*} \mathcal{J}_{n} \mathbb{T}\right.$ op $)$.

This functor has a left adjoint, given by applying $O(n) \wedge \Sigma_{n}$ - objectwise to an object of $\Sigma_{n} \ltimes\left(i^{*} \mathcal{J}_{n}\right.$ Top $):\left(O(n) \wedge \Sigma_{n} F\right)(U)=O(n) \wedge \Sigma_{n} F(U)$. The structure map is given below, where the first isomorphism is a standard result about group actions and the second is $O(n)_{+} \wedge \Sigma_{n}-$ applied to the structure map of $F$.

$$
O(n) \wedge_{\Sigma_{n}} F(U) \wedge \partial_{n}(U, V) \cong O(n) \wedge_{\Sigma_{n}}\left(F(U) \wedge i^{*} \mathcal{J}_{n}(U, V)\right) \rightarrow O(n) \wedge_{\Sigma_{n}} F(V)
$$

Just as with $\Sigma_{n} \ltimes\left(\mathcal{W}_{n}\right.$ Top $)$ and $O(n) \ltimes\left(\mathcal{J}_{n}\right.$ Top $)$, we can put an objectwise and an $n$-stable model structure on $\Sigma_{n} \ltimes\left(i^{*} \mathcal{J}_{n} \mathbb{T}\right.$ op $)$.

Lemma 5.19 The categories $\Sigma_{n} \ltimes\left(i^{*} \mathcal{J}_{n} \mathbb{T}\right.$ op $), O(n) \ltimes\left(\mathcal{J}_{n} \mathbb{T}\right.$ op) and $\Sigma_{n} \ltimes\left(\mathcal{W}_{n}\right.$ Top $)$ admit objectwise model structures where the weak equivalences and fibrations are those maps $f$ such that $f(A)$ is a weak equivalence or fibrations of based (non-equivariant) spaces for each $A$ in $i^{*} \mathcal{J}_{n}, \partial_{n}$ or $\mathcal{W}_{n}$. These model structures are cofibrantly generated and proper.

Proposition 5.20 Each of the categories $\Sigma_{n} \ltimes\left(i^{*} \mathcal{J}_{n} \mathbb{T}\right.$ op $), O(n) \ltimes\left(\mathcal{J}_{n} \mathbb{T}\right.$ op $)$ and $\Sigma_{n} \ltimes\left(\mathcal{W}_{n} \mathbb{T}\right.$ op $)$ admits an $n$-stable model structure which is the left Bousfield localisation of the objectwise model structure at the set of maps

$$
\begin{array}{rll}
i^{*} \mathcal{J}_{n}(U \oplus \mathbb{R},-) \wedge S^{n} \longrightarrow i^{*} \mathcal{J}_{n}(U,-) & \text { for } & \Sigma_{n} \ltimes\left(i^{*} \mathcal{J}_{n} \mathbb{T} \text { op }\right) \\
\mathcal{J}_{n}(U \oplus \mathbb{R},-) \wedge S^{n} \longrightarrow \mathcal{J}_{n}(U,-) & \text { for } & O(n) \ltimes\left(\mathcal{J}_{n} \mathbb{T o p}\right) \\
\mathcal{W}_{n}\left(X \wedge S^{1},-\right) \wedge S^{n} \longrightarrow \mathcal{W}_{n}(X,-) & \text { for } & \Sigma_{n} \ltimes\left(\mathcal{W}_{n} \mathbb{T} \text { op }\right)
\end{array}
$$


as $U$ (or $X)$ runs over the objects of a skeleton for $i^{*} \mathcal{J}_{n}\left(\right.$ or $\left.\mathcal{W}_{n}\right)$. The fibrant objects of $\Sigma_{n} \ltimes\left(i^{*} \mathcal{J}_{n}\right.$ Top) are those $E$ such that $E(V) \rightarrow \Omega^{n} E(V \oplus \mathbb{R})$ is a weak equivalence of spaces for all $V \in i^{*} \mathcal{J}_{n}$. Similar statements hold for $O(n) \ltimes\left(\mathcal{J}_{n} \mathbb{T}\right.$ op $)$ and $\Sigma_{n} \ltimes\left(\mathcal{W}_{n} \mathbb{T}\right.$ op $)$. These model structures are proper, stable and cofibrantly generated.

Proof. See [BE14, Proposition 4.12] and [BO13, Proposition 7.14] for $\Sigma_{n} \ltimes\left(\mathcal{W}_{n}\right.$ Top) and $O(n) \ltimes\left(\mathcal{J}_{n} \mathbb{T}\right.$ op $)$. The case of $\Sigma_{n} \ltimes\left(i^{*} \mathcal{J}_{n} \mathbb{T}\right.$ op $)$ is similar.

The weak equivalences of these categories are called $n \pi_{*}$-isomorphisms and are similar to $\pi_{*}$-isomorphisms of spectra. For example, let $F \in \Sigma_{n} \ltimes\left(\mathcal{W}_{n} \mathbb{T}\right.$ op $)$, then the $n \pi_{p}$-group of $F$ is defined in terms of the colimit of the system below.

$$
\pi_{p+n k}\left(F\left(S^{k}\right)\right) \longrightarrow \pi_{p+n k+1}\left(F\left(S^{k}\right) \wedge S^{n}\right) \longrightarrow \pi_{p+n k+1}\left(F\left(S^{k+1}\right)\right)
$$

For more details, see [BE14, Section 4.3] and [BO13, Section 7].

Lemma 5.21 The adjoint pair $\left(L_{n S}, n S^{*}\right)$ are a Quillen pair between the stable model structures on $\Sigma_{n} \ltimes\left(\mathcal{W}_{n} \mathbb{T}\right.$ op $)$ and $\Sigma_{n} \ltimes\left(i^{*} \mathcal{J}_{n} \mathbb{T}\right.$ op $)$.

Proof. It is clear that $n S^{*}$ preserves objectwise fibrations and weak equivalences. In BE14] the fibrant objects of the $n$-stable model structure on $\Sigma_{n} \ltimes\left(\mathcal{W}_{n}\right.$ Top $)$ are identified as those $F$ such that $F(A) \rightarrow \Omega^{n} F\left(A \wedge S^{1}\right)$ is a weak equivalence for all $A \in \mathcal{W}$. It is clear that for such an $F, S^{*} F$ is fibrant in $\Sigma_{n} \ltimes\left(i^{*} \mathcal{J}_{n}\right.$ Top $)$. It follows that we have a Quillen pair between the $n$-stable model structures.

Lemma 5.22 The functor $i^{*}$ is a right Quillen functor.

Proof. The functor $i^{*}$ preserves objectwise fibrations and weak equivalences. It is easy to check that it also preserves the weak equivalences of the $n$-stable model structure.

\subsection{Squares 1 and 2 commute}

We show that Squares 1 and 2 of Figure 4 (shown again below) commute. We have already defined the model categories and horizontal adjunctions, the vertical adjunctions are defined later in this section.

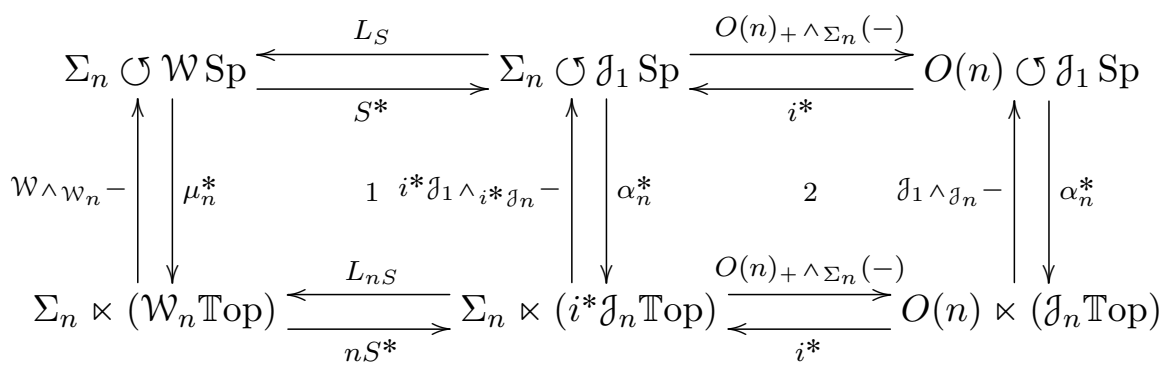


To prove the commutativity, we will make use of a commutative square of $\Sigma_{n} \circlearrowleft \mathbb{T}$ op-enriched categories. We will give the diagram first, then define the morphisms used.

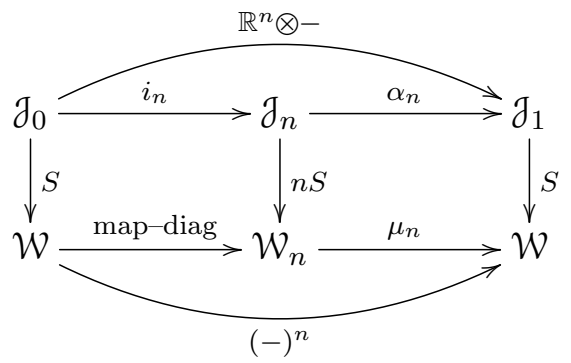

On objects the map $i_{n}: \mathcal{J}_{0} \rightarrow \mathcal{J}_{n}$ is the identity, on morphism spaces it is induced by the morphism $\mathrm{J}(U, V) \rightarrow \gamma_{n}(U, V)$ which sends $f$ to the pair $(f, 0)$.

The map $\alpha_{n}$ sends a vector space $U$ to $n U=\mathbb{R}^{n} \otimes U$. On morphism spaces $\alpha_{n}$ is induced by the map which takes $(f, x) \in \gamma_{n}(U, V)$ to $(n f, x) \in \gamma_{1}(n U, n V)$.

The morphism map-diag is the identity on objects and sends $\mathcal{W}(A, B)$ to $\mathcal{W}_{n}(A, B)$ by the diagonal. The morphism $\mu_{n}$ sends $A$ to $A^{\wedge n}$ and on morphism spaces acts as the smash product.

On objects, the diagram clearly commutes up to natural isomorphism. We must now show that the following diagram of morphism spaces commutes.

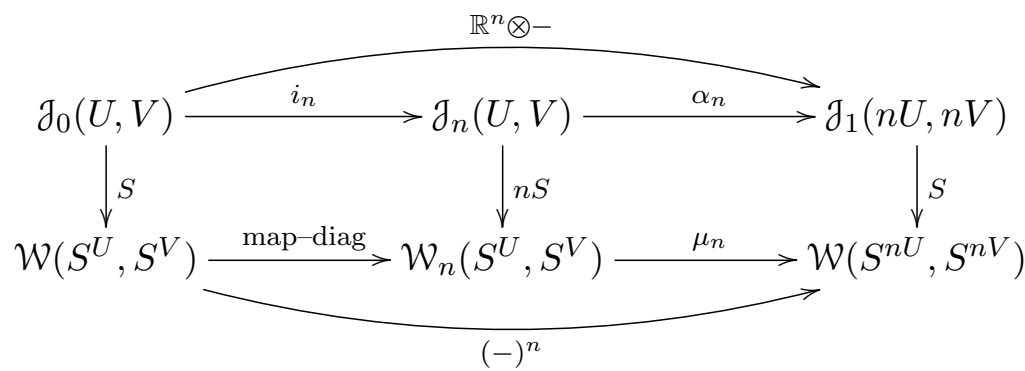

Starting with some map $f \in \mathcal{J}_{0}(U, V)$, it is easily checked that

$$
n S\left(i_{n} f\right)=(f, \ldots, f)=\operatorname{map}-\operatorname{diag}(S f)
$$

and hence the first square commutes. The second square is more complicated, but it suffices to consider a point of $(f, x) \in \gamma_{n}(U, V)$. We have the following equality of maps from $S^{\mathbb{R}^{n} \otimes U}$ to $S^{\mathbb{R}^{n} \otimes V}$, where $f: U \rightarrow V$ is an isometry and $x \in \mathbb{R}^{n} \otimes(V-f(U)$.

$$
S\left(\alpha_{n}(f, x)\right)=S((n f, x))
$$

The above terms are equal to the one-point compactification of the following map

$$
\begin{aligned}
\left(\mathbb{R}^{n} \otimes f\right)(-)+x: n U & \rightarrow n V \\
\left(u_{1} \oplus \cdots \oplus u_{n}\right) & \mapsto\left(f\left(u_{1}\right) \oplus \cdots \oplus f\left(u_{n}\right)\right)+x
\end{aligned}
$$


Now we consider the other direction around the square. Start with a pair $(f, x)$ as before, then we may write $x=x_{1} \oplus \cdots \oplus x_{n}$, using the projection of $\mathbb{R}^{n}$ onto its $n$ factors of $\mathbb{R}$. We then see that

$$
n S((f, x))=\left(S\left(f, x_{1}\right), \ldots, S\left(f, x_{n}\right)\right) \in \mathcal{W}_{n}\left(S^{U}, S^{V}\right)
$$

Applying $\mu_{n}$ to this element gives the map below.

$$
\left(u_{1} \oplus \cdots \oplus u_{n}\right) \mapsto f\left(u_{1}\right)+x_{1} \oplus \cdots \oplus f\left(u_{n}\right)+x_{n}
$$

It is now clear that the second square commutes.

We define the vertical adjunctions. The three pairs are defined similarly.

Definition 5.23 The functors

$$
\begin{aligned}
& \alpha_{n}^{*}: \Sigma_{n} \circlearrowleft \mathcal{J}_{1} \mathrm{Sp} \longrightarrow \Sigma_{n} \ltimes\left(i^{*} \mathcal{J}_{n} \mathbb{T} \mathrm{op}\right) \\
& \alpha_{n}^{*}: O(n) \circlearrowleft \mathcal{J}_{1} \mathrm{Sp} \longrightarrow O(n) \ltimes\left(\mathcal{J}_{n} \mathrm{Top}\right) \\
& \mu_{n}^{*}: \Sigma_{n} \circlearrowleft \mathcal{W} \mathrm{Sp} \longrightarrow \Sigma_{n} \ltimes\left(\mathcal{W}_{n} \mathbb{T o p}\right)
\end{aligned}
$$

are defined as pre-composition with $\alpha_{n}\left(\right.$ or $\left.\mu_{n}\right)$ along with a change of group action. For more details, see [BE14, Definition 5.1] and [BO13, Definition 8.2].

The functor $\alpha_{n}^{*}$ has a left adjoint, $\mathcal{J}_{1} \wedge \mathfrak{J}_{n}-$. On an object $E$ of $\Sigma_{n} \ltimes\left(i^{*} \mathcal{J}_{n}\right.$ Top $)$ it is given by the formula

$$
\left(\mathcal{J}_{1} \wedge \mathcal{J}_{n}-E\right)(V)=\int^{U \in \mathcal{J}_{n}} E(U) \wedge \mathcal{J}_{1}(U, n V) .
$$

We let $O(n)$ act on $n V=\mathbb{R}^{n} \otimes V$ via the standard action on $\mathbb{R}^{n}$. This induces an action on $\mathcal{J}_{1}(U, n V)$ and we let $O(n)$ act diagonally on the smash product $E(U) \wedge \mathcal{J}_{1}(U, n V)$. Equally we can define $i^{*} \mathcal{J}_{1} \wedge_{i}^{*} \mathcal{J}_{n}-$. The functor $\mu_{n}^{*}$ has a left adjoint $\mathcal{W}_{n} \wedge \mathcal{W}-$ given by a similar formula.

Proposition 5.24 The adjoint pairs $\left(\mathcal{W}_{n} \wedge \mathcal{W}-, \mu_{n}^{*}\right)$ and $\left(i^{*} \mathcal{J}_{1} \wedge_{i * J_{n}}-, \alpha_{n}^{*}\right)$ are Quillen equivalences.

Proof. The first adjunction is a Quillen pair by [BE14, Proposition 5.4]. The second by a similar argument as in [BO13, Section 8].

Proposition 5.25 The square labelled 1 in Figure 4 (displayed at the beginning of this section) commutes up to natural isomorphism: $\alpha_{n}^{*} \circ S^{*} \cong n S^{*} \circ \mu_{n}^{*}$. The square of left adjoints also commutes.

Proof. We have shown above that $S \circ \alpha_{n}=\mu_{n} \circ n S$. Since the right adjoints are all defined in terms of pre-composition the result follows. We note that $\alpha_{n}$ and $\mu_{n}$ also change the way the group $\Sigma_{n}$ acts, but it is easily checked that they do so in compatible ways.

Corollary 5.26 The adjoint pair $\left(L_{n S}, n S^{*}\right)$ is a Quillen equivalence.

Proof. This is a Quillen pair by Lemma 5.21. The other adjunctions in the square are Quillen equivalences, see Lemma 5.14, Proposition 5.24 and [BE14, Proposition 5.4]. Hence this Quillen pair is a Quillen equivalence. 
Lemma 5.27 The square labelled 2 in Figure 4 (also displayed at the beginning of this section) commutes up to natural isomorphism. That is, $\alpha_{n}^{*} \circ i^{*} \cong i^{*} \circ \alpha_{n}^{*}$. The square of left adjoints also commutes.

Proof. Let $E \in O(n) \mathcal{J}_{1} \operatorname{Sp}$. Then $\left(i^{*} \alpha_{n}^{*} E\right)(V)=i^{*} E(n V)$, where we have altered the action to involve the action of $O(n)$ on $n V$. We also have $\left(\alpha_{n}^{*} i^{*} E\right)(V)=i^{*} E(n V)$, with the action altered similarly.

\subsection{Square 3 commutes}

Now we can describe the extent to which the square below (labelled 3 in Figure 4 ) commutes. Recall that $\operatorname{Res}_{0}^{n}:=i_{n}^{*}$ and $\operatorname{Ind}_{0}^{n}$ is the $n^{t h}$ derivative (Definition 2.15).

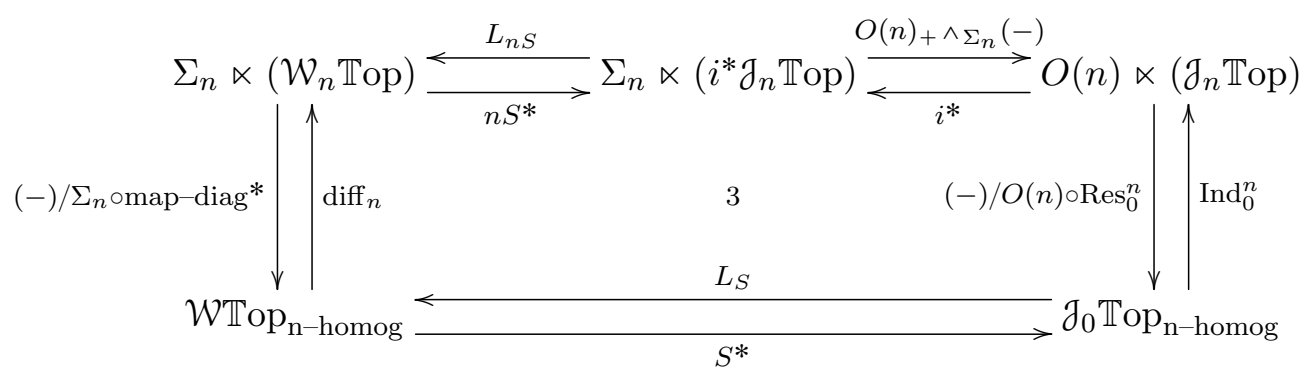

We claim that the following composite functors agree up to natural isomorphism.

$$
(-) / O(n) \circ \operatorname{Res}_{0}^{n} \circ O(n)_{+} \wedge_{\Sigma_{n}}(-) \circ n S^{*} \cong S^{*} \circ(-) / \Sigma_{n} \circ \operatorname{map}-\operatorname{diag}^{*}
$$

Remark 5.28 Unfortunately, these composites consist of both left and right Quillen functors. So it will not follow automatically that we have a commuting square on the level of homotopy categories. Thus we will delay homotopical considerations until after we have proven the claimed commutativity.

The key fact comes from the commuting squares of diagram categories of Section 5.4 .

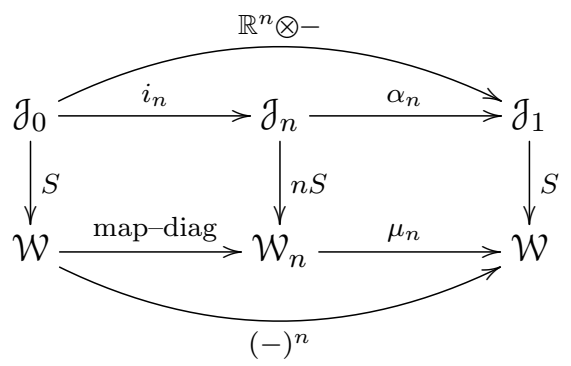

The left hand square of this diagram tells us that $\operatorname{Res}_{0}^{n} \circ n S^{*}=S^{*} \circ$ map-diag* We also know that for any $\Sigma_{n}$-space $X$,

$$
\left(O(n)_{+} \wedge \Sigma_{n} X\right) / O(n) \cong X / \Sigma_{n}
$$


and that this isomorphism is natural in $X$. Hence, for any $E$ in $\Sigma_{n} \ltimes\left(\mathcal{W}_{n} \mathbb{T}\right.$ op $)$ there are natural isomorphisms

$$
\begin{aligned}
\left(\operatorname{Res}_{0}^{n}\left(O(n)_{+} \wedge \Sigma_{n}\left(n S^{*} E\right)\right) / O(n)\right. & =\left(\left(O(n)_{+} \wedge \Sigma_{n}\left(E \circ n S \circ i_{n}\right)\right) / O(n)\right. \\
& \cong\left(E \circ n S \circ i_{n}\right) / \Sigma_{n} \\
& \cong(E \circ \operatorname{map}-\operatorname{diag} \circ S) / \Sigma_{n} \\
& =S^{*}\left(\left(\operatorname{map}-\operatorname{diag}^{*} E\right) / \Sigma_{n}\right)
\end{aligned}
$$

Thus, square 3 commutes up to natural isomorphism.

As already noted, $S^{*}$ and $n S^{*}$ are right Quillen functors while $(-) / \Sigma_{n}$ and $(-) / O(n)$ are left Quillen functors. We must then look a little deeper to find a homotopically meaningful description of how these functors (or rather, their derived counterparts) commute.

Proposition 5.29 The following diagram of homotopy categories commutes up to natural isomorphism. We use $L$ and $R$ to indicate where we have taken left or right derived functors.

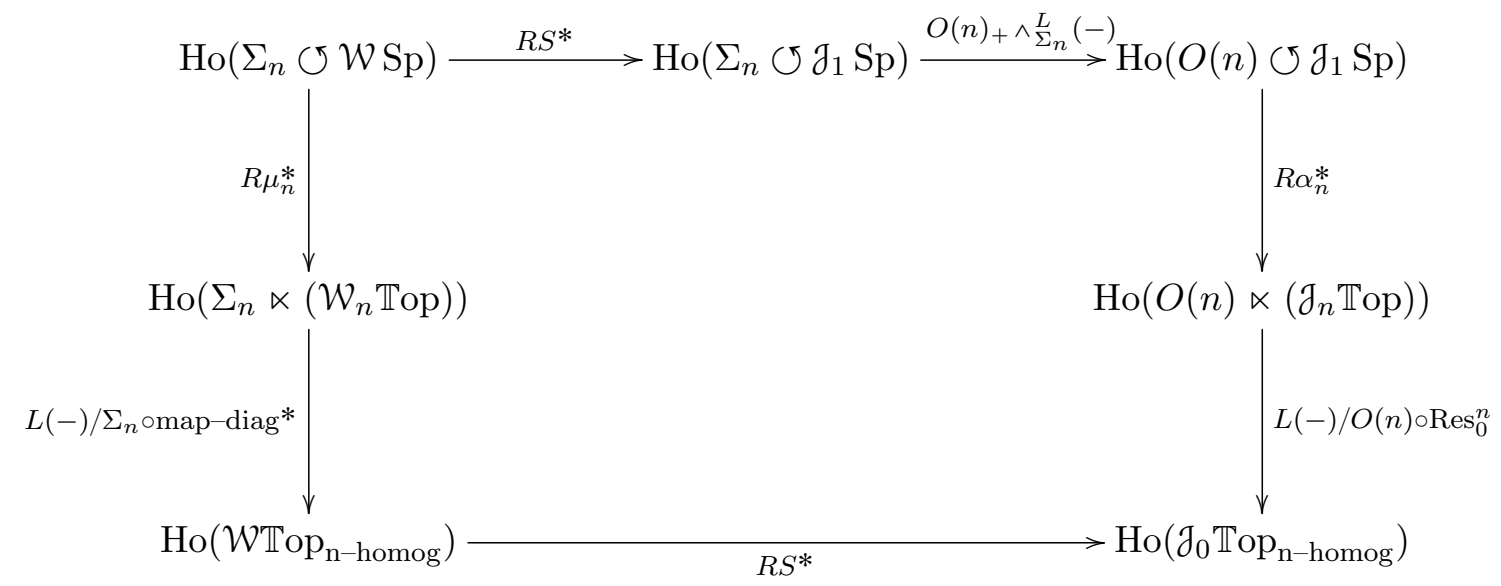

Proof. By [BE14, Theorem 6.6] the composite of $R \mu_{n}^{*}$ and $L(-) / \Sigma_{n} \circ$ map-diag* applied to a $\Sigma_{n}$-spectrum $\Theta$ is weakly equivalent to the formula

$$
X \mapsto \Omega^{\infty}\left(\left(\Theta \wedge A^{\wedge n}\right) / h \Sigma_{n}\right)
$$

of Theorem 2.13. Similarly, by [BO13, Theorem 10.1] the composite of $R \alpha_{n}^{*}$ and $L(-) / O(n) \circ$ $\operatorname{Res}_{0}^{n}$ applied to an $O(n)$-spectrum $\Psi$ is weakly equivalent to the formula

$$
V \mapsto \Omega^{\infty}\left(\left(\Psi_{E} \wedge S^{\mathbb{R}^{n} \otimes V}\right) / h O(n)\right)
$$

of Theorem 2.13. The result then follows by Proposition 3.1.

Theorem 5.30 The following square commutes up to natural isomorphism.

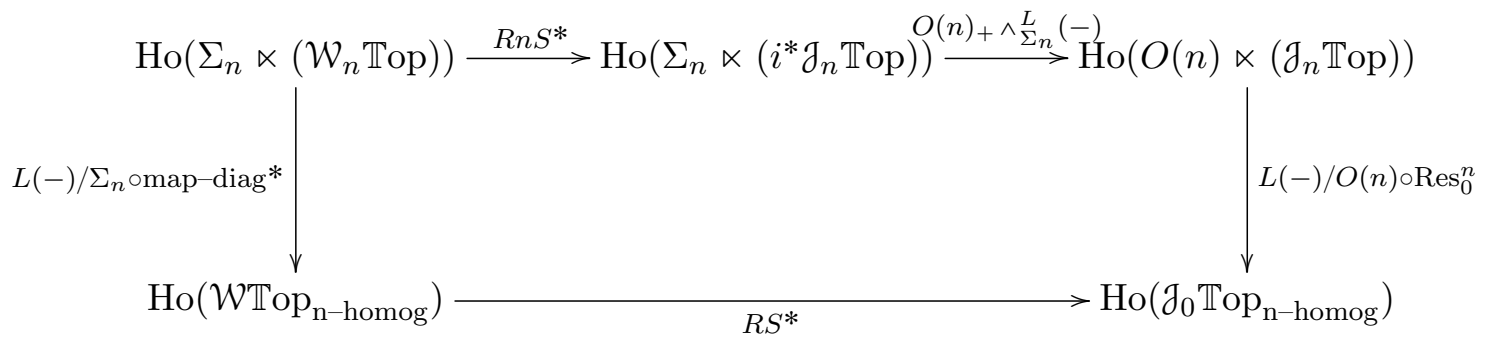


Proof. Take some $E \in \Sigma_{n} \ltimes\left(\mathcal{W}_{n} \mathbb{T}\right.$ op $)$. Since we are interested in the homotopy category, this is the same as choosing some spectrum $\Theta$ with a $\Sigma_{n}$-action. By Proposition 5.29 the two images of $\Theta$ in $\operatorname{Ho}\left(\mathcal{J}_{0} \mathbb{T}_{0} p_{n-h o m o g}\right)$ agree. This, combined with the commutativity of Squares 1 and 2 (on the level of homotopy categories) completes the proof.

\section{References}

[ADL08] G. Arone, W. Dwyer, and K. Lesh. Loop structures in Taylor towers. Algebraic and Geometric Topology, 8:173-210, 2008.

[Aro02] G. Arone. The Weiss derivatives of BO (-) and BU (-). Topology, 41(3):451-481, 2002.

[BCR07] G. Biedermann, B. Chorny, and O. Röndigs. Calculus of functors and model categories. Adv. Math., 214(1):92-115, 2007.

[BE14] D. Barnes and R. Eldred. Capturing Goodwillie's derivative. arXiv: 1406.0424 , 2014.

[BO13] D. Barnes and P. Oman. Model categories for orthogonal calculus. Algebr. Geom. Topol., 13(2):959-999, 2013.

[BR13] G. Biedermann and O. Röndigs. Calculus of functors and model categories II. arXiv: $1305: 2834,2013$.

[Goo90] T. Goodwillie. Calculus I: The first derivative of pseudoisotopy theory. K-Theory, 4(1):1-27, 1990.

[Goo91] T. Goodwillie. Calculus II: Analytic functors. K-Theory, 5(4):295-332, 1991.

[Goo03] T. Goodwillie. Calculus III: Taylor Series. Geom. Topol., 7:645-711, 2003.

[Hir03] P. Hirschhorn. Model categories and their localizations, volume 99 of Mathematical Surveys and Monographs. American Mathematical Society, Providence, RI, 2003.

[Hus94] D. Husemoller. Fibre bundles, volume 20 of Graduate Texts in Mathematics. Springer-Verlag, New York, third edition, 1994.

[MM02] M. Mandell and J. May. Equivariant orthogonal spectra and S-modules. Mem. Amer. Math. Soc., 159(755):x+108, 2002.

[MMSS01] M. Mandell, J. May, S. Schwede, and B. Shipley. Model categories of diagram spectra. Proc. London Math. Soc. (3), 82(2):441-512, 2001.

[RW15] R. Reis and M. Weiss. Functor calculus and the discriminant method (smooth maps to the plane and pontryagin classes, Part III). arXiv:1102.0233v5, 2011 (v3 2015).

[Wei95] M. Weiss. Orthogonal calculus. Trans. Amer. Math. Soc., 347(10):3743-3796, 1995. 
[Wei98] M. Weiss. Erratum: "Orthogonal calculus" [Trans. Amer. Math. Soc. 347(10):3743-3796, 1995 ]. Trans. Amer. Math. Soc., 350(2):851-855, 1998.

David Barnes

Pure Mathematics Research Centre, Queen's University, Belfast BT7 1NN, UK d.barnes@qub.ac.uk
Rosona Eldred

Mathematisches Institut, Universität Münster Einsteinstr. 62, 48149 Münster, Germany eldred@uni-muenster.de 